\title{
On the Physical Interpretation of Density Operators at the Atomic Scale: A Thorough Analysis of Some Simple Cases
}

\author{
JUAN C. PANIAGUA
}

Departament de Química Física, Universitat de Barcelona, C. Martí i Franquès 1, E-08028 Barcelona, Spain

\begin{abstract}
The physical meaning of the concepts underlying the density operator formalism are analyzed from different points of view. In particular, the diversity of ways of expressing the density operator of the simplest NMR sample is exploited to show that there are many molecular-scale physical pictures compatible with each macroscopic state, some of them being more useful than others for specific aims. Those corresponding to diagonal representations of the density matrix conform closely to classical-like pictures, which allow us to ignore the subtle effects of quantum interferences that are implicit in the concept of coherence. A widespread biconical picture that does not rely on a sound physical basis is shown to be quantitatively valid provided that ad hoc populations are chosen for the involved quantum states. The interpretation of the coefficients of the density operator expansion in terms of observable-related basis elements is discussed to show that the identification of these with the corresponding physical properties can be misleading in certain cases. (c) 2006 Wiley Periodicals, Inc. Concepts Magn Reson Part A 28A: 384-409, 2006
\end{abstract}

KEY WORDS: density operator; density matrix; coherences; phase coherence; physical interpretation

\section{INTRODUCTION}

The density operator formalism plays a central role in the formulation of nuclear magnetic resonance, while many other spectroscopic techniques can be satisfac-

Received 3 July 2006; revised 21 August 2006; accepted 21 August 2006

Correspondence to: Juan C. Paniagua; E-mail: jpaniagua @ub.edu Concepts in Magnetic Resonance Part A, Vol. 28A(6) 384-409 (2006) Published online in Wiley InterScience (www.interscience.wiley. com). DOI 10.1002/cmr.a.20070

๑) 2006 Wiley Periodicals, Inc. torily explained by referring only to wavefunctions. The fact that most chemistry undergraduate curricula do not include that formalism, together with the mathematical baggage necessary to fully understand it and the subtleties of its physical meaning, may obscure the interpretation of what is going on at the atomic scale in the experiments. This puts in doubt the validity of certain intuitive reasonings that are useful for making easier the application of the rules determining the spin evolution in NMR experiments.

For instance, the density operator that describes a sample with molecules having one proton each at thermodynamic equilibrium under a static magnetic 
field is normally expressed as a mixture of the two $\hat{I}_{z}$ eigenstates, $\Phi_{\alpha}$ and $\Phi_{\beta}$. This suggests a physical picture in which somewhat more than one half of the spins have $I_{z}=\hbar / 2$ and the rest have $I_{z}=-\hbar / 2$, but this image is unsatisfactory from the physical point of view. In effect, in an isolated sample at equilibrium, all directions are equivalent, so that one would expect that the proton spins were oriented at random. Let us assume that a magnetic field is gradually introduced. When this begins to act, it is hard to believe that a very small field intensity produces a sudden reorientation of every spin so that its expectation value becomes aligned with the $z$ axis. One would rather expect that the initial randomly oriented distribution of spins be piecemeal distorted in such a way that the population becomes larger the closer the spin orientations are to the field direction. This microscopic picture looks rather different from the above-mentioned binary mixture of $\Phi_{\alpha}$ and $\Phi_{\beta}$ states, and it is seldom considered in textbooks, a notable exception being the excellent text of M. H. Levitt (1). To put that picture into a quantitative basis we have to know the population density for an arbitrary spin orientation. This is not a trivial matter because the Boltzmann distribution formula can only be applied to a distribution of energy eigenstates.

An alternative picture is often used in which the spins are distributed among two opposite cones that precess around a magnetic field with randomly distributes phases. This is combined with intuitive ideas of precession phase grouping under the effect of a pulse. Although such arguments are useful for interpreting the concept of "coherence," they are often used with mistrust because that phase grouping is not based on physically sound arguments.

In this article, we explore what can be said about the behavior of an NMR sample at the atomic scale by reflecting on the physical significance of the density operator and exploiting the fact that different samples, prepared using different procedures, can have the same density operator and, therefore, the same behavior. This makes them indistinguishable, while it provides alternative pictures that can be useful for different aims. Several interpretations of the density operator of a simple NMR sample that are often used on an intuitive basis are formalized, and the validity of different classical-like pictures is discussed. The physical meaning of coherences is analyzed from several points of view, paying attention to the fact that diagonal (coherence-free) representations can always be chosen that lead to pictures closest to the classical view. The widespread interpretation based on the above-mentioned biconical picture is shown to lead to valid conclusions despite being grounded on unphysi- cal arguments. The concept of "phase coherence," often associated with this image, is seen to have a clearer meaning when interpreted within a more natural picture.

The interpretation of the expansion coefficients of the density operator in terms of observable-related operators is also discussed, and the identification of the operators appearing in the expansion with expectation values of the corresponding observables is shown to be misleading in certain cases. Finally, comparison with other spectroscopic techniques is made to throw some light on the differences of the formalisms normally used in different contexts. Although part of the material presented can be found in standard quantum physics and NMR texts, we have thought it worthwhile to include it to make the exposition self-contained.

\section{PURE AND MIXED STATES}

In the most extended Copenhagen interpretation of quantum mechanics, to which we stick from now on, it is postulated that the maximum information that can be known on the state of a physical system is embodied in a state vector or wavefunction $\Psi$ (we identify both concepts although, strictly speaking, the latter is a particular way of representing the former). The probabilistic nature of the theory limits this information to probabilities for different outcomes of measurements, average values over a large number of identical systems (expectation values), and other probability distribution moments. All these data are extracted from the wave function by evaluating the expression

$$
\langle A\rangle_{\Psi}=\langle\Psi|\hat{A}| \Psi\rangle
$$

for a suitably chosen Hermitian operator $\hat{A}$. While no measurement is being made on the system its evolution is determined by the time-dependent Schrödinger equation

$$
i \hbar \frac{\partial \Psi}{\partial t}=\hat{H} \Psi
$$

where $\hat{H}$ - the Hamiltonian operator-is the quantum operator associated to the total energy. Hence, the knowledge of the energy components relevant for the system together with the wavefunction at a time $t_{0}$, allows - through the integration of that differential equation-us to calculate the wavefunctions at any later time $t$. This evolution is often expressed by 
introducing an evolution operator $\hat{U}\left(t, t_{0}\right)$ that renders $\Psi(t)$ when applied to $\Psi\left(t_{0}\right)$

$$
\hat{U}\left(t, t_{0}\right) \Psi\left(t_{0}\right)=\Psi(t) .
$$

For a time-independent Hamiltonian $\left(\widehat{H_{0}}\right)$ substitution of this equation into Eq. [2] leads to

$$
\hat{U}\left(t, t_{0}\right)=e^{-i\left(t-t_{0}\right) \widehat{H_{0} / \hbar}} .
$$

The preceding formalism is adequate to deal with simple atomic scale systems as long as one can conceive experiences that provide the necessary information for specifying their wavefunctions. However, it is not feasible to determine the wavefunction of, say, every molecule in a macroscopic gaseous sample, in the same way that one cannot aim at knowing their classical trajectories. Moreover, wavefunctions are often not known even in atomic scale experiments. The measurements that can be performed on a macroscopic sample normally provide average values over a large number of atomic scale systems, as happens with the temperature of a gas or the magnetization of an NMR sample. Although these values do not permit to specify individual molecular wavefunctions, they furnish a probabilistic information that, together with a atomic scale model of the sample, allows us to predict the evolution of macroscopic properties.

Let us consider a fluid sample made of independent or weakly interacting atomic scale systems, so that an individual physical state can be attached to each of them. We refer to them as "molecules," although they could also be, for instance, sets of coupled nuclear spins within a molecule. If the probabilities for any molecule to be in each one of a set of states described by the wavefunctions $\Psi_{1}, \ldots \Psi_{n}$ are $p_{1}, \ldots p_{n}$, we say that the molecules of the sample are in a statistical mixture of the pure states $\Psi_{1}, \ldots \Psi_{n}$, weighted with the coefficients $p_{1}, \ldots p_{n}$. Although, for simplicity, we are assuming that these wavefunctions form a discrete set, this is not a necessary restriction, and we later extend the following expression to the continuous case. Every mixture of pure states-also referred to as a mixed state-is represented by a density or statistical operator defined by

$$
\hat{\rho} \equiv \sum_{k=1}^{n} p_{k}\left|\Psi_{k}\right\rangle\left\langle\Psi_{k}\right|
$$

where $\left|\Psi_{k}\right\rangle\left\langle\Psi_{k}\right|$ is an operator that, when applied to any wavefunction $f$, projects it onto the direction defined by the vector $\Psi_{k}$ in the molecular Hilbert space:

$$
\left|\Psi_{k}\right\rangle\left\langle\Psi_{k}\right| f=\Psi_{k}\left\langle\Psi_{k} \mid f\right\rangle .
$$

Because the number $N$ of molecules in a macroscopic sample is large, the probability $p_{k}$ of a molecule being in a pure state $\Psi_{k}$ multiplied by $N$ gives, to a good approximation, the number of molecules in that state $\left(N_{k}\right)$; that is, the population of that state. So, $p_{k}=N_{k} / N$ is also the normalized population-for short, the population-of the state $\Psi_{k}$.

A density operator contains the physical information of a mixed state in much the same way as a wavefunction determines a pure state. Each piece of physical information of the mixed state is an average over the wavefunctions composing the mixture

$$
\langle A\rangle_{\rho}=\sum_{k=1}^{n} p_{k}\left\langle\Psi_{k}|\hat{A}| \Psi_{k}\right\rangle
$$

and can be expressed in the form

$$
\langle A\rangle_{\rho}=\sum_{k=1}^{n} p_{k} \operatorname{tr}\left[\left|\Psi_{k}\right\rangle\left\langle\Psi_{k}\right| \hat{A}\right]=\operatorname{tr}(\hat{\rho} \hat{A}) .
$$

If $p_{k}=1$ for some state $\Psi_{k}$ (in which case $p_{l}$ must vanish for any $l \neq k$ ), the state represented by the density operator

$$
\hat{\rho}_{k}=\left|\Psi_{k}\right\rangle\left\langle\Psi_{k}\right|
$$

is pure, and only in this case we can attach a wavefunction to it. Equations [7] and [8] then reduce to Eq. [1].

By applying the time-dependent Schrödinger equation to each pure state of a statistical mixture, one readily obtains the evolution equation for the corresponding density operator, which is known as the Liouville-von Neumann equation:

$$
i \hbar \frac{d \hat{\rho}}{d t}=[\hat{H}, \hat{\rho}] .
$$

Its solution can be expressed in terms of the time evolution operator (Eq. [3]):

$$
\begin{aligned}
\hat{\rho}(t) & =\sum_{k=1}^{n} p_{k}\left|\Psi_{k}(t)\right\rangle\left\langle\Psi_{k}(t)\left|=\sum_{k=1}^{n} p_{k}\right| \hat{U}\left(t, t_{0}\right) \Psi\left(t_{0}\right)\right\rangle \\
& \cdot\left\langle\hat{U}\left(t, t_{0}\right) \Psi\left(t_{0}\right)\right|=\hat{U}\left(t, t_{0}\right) \hat{\rho}\left(t_{0}\right) \hat{U}^{\dagger}\left(t, t_{0}\right) . \quad
\end{aligned}
$$




\section{DENSITY MATRICES}

Let us assume that the Hilbert space formed by the molecular wavefunctions is $m$ dimensional. If we choose an orthonormal basis $\left\{\Phi_{1}, \ldots \Phi_{m}\right\}$ in this space we can represent each wavefunction $\Psi_{k}$ by a one-column matrix $\left(\begin{array}{c}c_{1 k} \\ \cdots \\ c_{m k}\end{array}\right)$ whose elements are the components of $\Psi_{k}$ on every basis function:

$$
\Psi_{k}=\sum_{i=1}^{m} c_{i k} \Phi_{i}
$$

By introducing this development in Eq. [9],

$$
\hat{\boldsymbol{\rho}}_{k}=\left|\sum_{i=1}^{m} c_{i k} \Phi_{i}\right\rangle\left\langle\sum_{j=1}^{m} c_{j k} \Phi_{j}\left|=\sum_{i=1}^{m} \sum_{j=1}^{m} c_{i k} c_{j k}^{*}\right| \Phi_{i}\right\rangle\left\langle\Phi_{j}\right|,
$$

and putting this result into the definition of $\hat{\rho}$ (Eq. [5]), we obtain

$$
\hat{\rho}=\sum_{i=1}^{m} \sum_{j=1}^{m} \sum_{k=1}^{n} p_{k} c_{i k} c_{j k}^{*}\left|\Phi_{i}\right\rangle\left\langle\Phi_{j}\left|=\sum_{i=1}^{m} \sum_{j=1}^{m} \rho_{i j}\right| \Phi_{i}\right\rangle\left\langle\Phi_{j}\right|,
$$

where

$$
\rho_{i j} \equiv \sum_{k=1}^{n} p_{k} c_{i k} c_{j k}^{*}=\left\langle\Phi_{i}|\hat{\rho}| \Phi_{j}\right\rangle
$$

are the elements of an $m \times m$ matrix: the density matrix ( $\boldsymbol{\rho}$ ) that represents the density operator $\hat{\rho}$ in the wavefunction basis $\left\{\Phi_{1}, \ldots \Phi_{m}\right\}$ :

$$
\boldsymbol{\rho}=\left(\begin{array}{lll}
\rho_{11} & \cdots & \rho_{1 m} \\
\cdots & \cdots & \cdots \\
\rho_{m 1} & \cdots & \rho_{m m}
\end{array}\right)
$$

Contrary to what happens with the density operator definition (Eq. [5]), the expansion [13] has, in general, cross-terms: $\rho_{i j}\left|\Phi_{i}\right\rangle\left\langle\Phi_{j}\right|$ with $i \neq j$. These terms, as well as the corresponding nondiagonal elements of the density matrix $\left(\rho_{i j}\right)$, are known as coherences. It is said that $\rho_{i j}$ is a coherence between the pure states $\Phi_{i}$ and $\Phi_{j}$.

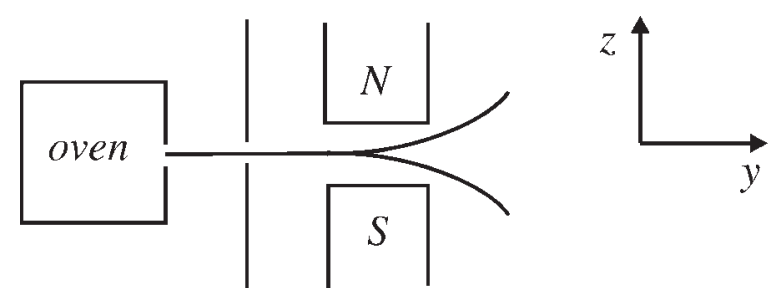

Figure 1 A Stern-Gerlach device oriented so as to measure the spin $z$ component of the particles emerging from an oven. The magnetic field has a gradient along the $z$ axis.

\section{THE MEANING OF POPULATIONS}

In the text preceding Eq. [5] we have introduced the population of a pure state in a statistical mixture as the probability of a molecule to be in that state. In principle, such probabilities could be known by making the appropriate measurements.

As an example, consider an oven with many identical $\frac{1}{2}$-spin particles that can escape through a hole oriented along the $y$ Cartesian axis (Fig. 1). The emerging particles enter a Stern-Gerlach apparatus that measures their spin $z$ component. If the particles are produced in isotropic conditions, the device will split the beam into two equally populated parts with spin $z$-components $\hbar / 2$ and $-\hbar / 2$, respectively. Thus, the density operator describing the spin state of the particles emerging from the oven is

$$
\hat{\rho}=\frac{1}{2}\left|\Phi_{\alpha}\right\rangle\left\langle\Phi_{\alpha} \mid+\frac{1}{2} \Phi_{\beta}\right\rangle\left\langle\Phi_{\beta}\right|,
$$

where the projection operators $\left|\Phi_{\alpha}\right\rangle\left\langle\Phi_{\alpha}\right|$ and $\left|\Phi_{\beta}\right\rangle\left\langle\Phi_{\beta}\right|$ represent pure spin states in which the spin $z$ component has the values $\hbar / 2$ (wavefunction $\Phi_{\alpha}$ ) and $-\hbar / 2$ (wavefunction $\Phi_{\beta}$ ), respectively.

It could be concluded from this result that half of the particles actually emerged from the oven in the state $\Phi_{\alpha}$ and the other half did it in the state $\Phi_{\beta}$. But we could have oriented the Stern-Gerlach device in any direction perpendicular to the $y$ axis and the result would be, again, a splitting of the beam in two parts with equal numbers of particles. For instance, if the device were rotated $90^{\circ}$ so as to measure the $x$ component of the spin, the results would suggest that half of the particles would enter the magnetic field with a well-defined positive $x$ component and the other half would do with a negative one. The density operator representing this situation is

$$
\hat{\rho}=\frac{1}{2}\left|\Phi_{+x}\right\rangle\left\langle\Phi_{+x}\left|+\frac{1}{2}\right| \Phi_{-x}\right\rangle\left\langle\Phi_{-x}\right|
$$


where $\Phi_{+x}$ and $\Phi_{-x}$ are pure states with a welldefined spin $x$ component of $+\hbar / 2$ and $-\hbar / 2$, respectively.

Which of these two density operators should we use for describing the particles emerging from the oven? This question is irrelevant, as both expressions are different representations of the same operator and, therefore, they lead to the same probability distributions for every observable. This mathematical equivalency is readily verified by expressing $\Phi_{ \pm x}$ in terms of $\Phi_{\alpha}$ and $\Phi_{\beta}$ :

$$
\Phi_{ \pm x}=\frac{1}{\sqrt{2}}\left(\Phi_{\alpha} \pm \Phi_{\beta}\right),
$$

and substituting into Eq. [17]

$$
\begin{aligned}
\hat{\rho}=\frac{1}{4}\left|\Phi_{\alpha}+\Phi_{\beta}\right\rangle\left\langle\Phi_{\alpha}+\Phi_{\beta}\left|+\frac{1}{4}\right| \Phi_{\alpha}-\Phi_{\beta}\right\rangle \\
\cdot\left\langle\Phi_{\alpha}-\Phi_{\beta}\left|=\frac{1}{2}\right| \Phi_{\alpha}\right\rangle\left\langle\Phi_{\alpha}\left|+\frac{1}{2}\right| \Phi_{\beta}\right\rangle\left\langle\Phi_{\beta}\right| \cdot
\end{aligned}
$$

Yet the physical pictures suggested by the expressions [16] and [17] are incompatible: a spin cannot have well-defined components along two different axes. Thus, we cannot say that half of the particles have $I_{z}=\hbar / 2$ and the other half have $I_{z}=-\hbar / 2$ while they also possess the values $\pm \hbar / 2$ of $I_{x}$. In fact, it can be shown that every pure spin state of a $\frac{1}{2}$-spin particle has a well-defined spin component along one-and only one-axis. Likewise, this is the direction of the average spin vector, which, for brevity's sake, is referred to as the "spin orientation." We also say that the spin vector "points" to that direction.

One could argue that the Stern-Gerlach device introduces a directionality that could align the spin of the particles inside the oven along the magnetic field gradient direction. Their states would then be $\Phi_{\alpha}$ or $\Phi_{\beta}$ if the device is settled so as to measure the $z$ spin component and $\Phi_{+x}$ or $\Phi_{-x}$ if it is oriented along the $x$ axis. However, this possibility has been ruled out by experiments in which the direction along which the spin is going to be measured is chosen after the particle has abandoned the oven (2).

The fact that no wavefunction nor density operator determines the spin components along two or three different axes could suggest that those are not convenient mathematical tools to describe the true spin state of the particles. However, a parallel discussion with $\frac{3}{2}$-spin particles shows that the impossibility of different spin components being defined at the same time is not a flaw of the theory but a feature of nature itself.
In effect, the values that can be obtained in a measurement of any spin component are now $\pm \hbar / 2$ and $\pm 3 \hbar / 2$. Hence, the square of any component can only take the values $\hbar^{2} / 4$ and $9 \hbar^{2} / 4$, and there is no sum of three such values that equals the squared spin module, which has a well-defined value of $s(s+1) \hbar^{2}=$ $15 \hbar^{2} / 4$. That is, the three spin components cannot simultaneously have well-defined values. When the spin component along one axis is measured, any previous information about components along perpendicular axes is erased. A digital camera that allows us to register only one picture provides an illustrating analogy: if after having taken a picture of, say, a vertical pole we photograph a horizontal one, the first image is lost.

The above discussion makes clear that the outcomes of the measurements of spin components do not allow us to univocally assign a pure-state population distribution to the particles in the oven. In fact, we have seen in section 3 that a density operator is represented by a different density matrix in every orthonormal basis of the wavefunction space, and the diagonal elements of any such matrix represent populations of the corresponding basis vectors. In effect, the probability $P_{\rho}\left(\Phi_{i}\right)$ of finding the pure state $\Phi_{i}$ in the mixture [5] is the expectation value of the projector $\left|\Phi_{i}\right\rangle\left\langle\Phi_{i}\right|$; that is (see Eq. [13]):

$$
P_{\rho}\left(\Phi_{i}\right)=\operatorname{tr}\left(\hat{\rho}\left|\Phi_{i}\right\rangle\left\langle\Phi_{i}\right|\right)=\left\langle\Phi_{i} \mid \hat{\rho} \Phi_{i}\right\rangle=\rho_{i i} .
$$

But what do we mean with the probability of "finding" the pure state $\Phi_{i}$ in a mixture? This is a short way of referring to the probability of obtaining values that characterize univocally the state $\Phi_{i}$ when the convenient measurements are made, which does not imply that $\Phi_{i}$ was the wavefunction describing the system before the measurement.

Thus, if $\Phi_{i}$ is an eigenfunction with a nondegenerate eigenvalue $a_{i}$ of the operator $\hat{A}$ associated to a one-particle observable $A$, a measurement of this that filters that value selects particles in the pure state $\Phi_{i}$. This measurement process changes any previous particle state to $\Phi_{i}$, so we cannot say that this was the state of the filtered particles before they entered the measurement device. Moreover, if we would have decided to measure another observable $A^{\prime}$ whose eigenvalues characterize a basis set $\left\{\Phi_{i}^{\prime}\right\}$ not including $\Phi_{i}$, this state could never be found. Hence, the multiplicity of equivalent population distributions does not entail any contradiction.

Before going on, it is worthwhile to stress the difference between a statistical mixture and a pure state leading to the same probabilities for some observable. The wave functions $\Phi_{ \pm x}$, for instance, are 
linear combinations or "superpositions" of $\Phi_{\alpha}$ and $\Phi_{\beta}$ (see Eq. [18]) that lead to the same probabilities for the outcomes $\pm \hbar / 2$ in a measurement of $I_{z}$, as does the statistical mixture of $\Phi_{\alpha}$ and $\Phi_{\alpha}$ defined by the density operator [16]. But those wavefunctions contain a more precise information than this operator. Thus, the result of a measurement of $I_{x}$ is predetermined in any of the pure states [18] but not in the mixed state [16]. In fact, there is an infinity of pure states of the form

$$
\Phi_{\varphi}=\frac{1}{\sqrt{2}}\left(\Phi_{\alpha}+e^{i \varphi} \Phi_{\beta}\right) \quad \text { with } \varphi \in[0,2 \pi),
$$

leading to the same probability distribution for $I_{z}$. For each of them the average spin vector points along some direction in the $x y$ plane, while no directionality exists in the state [16].

The preceding discussion can also be applied to an NMR sample whose molecules contain one proton each — so that the protons interact only weakly among themselves at thermodynamic equilibrium in the absence of external fields; that is, before putting it into the magnet (although in the case of the oven we have only considered particles emerging along the $y$ axis, the hole could have been done at any point of the oven wall, so that the conclusions can be extended to particles moving along any direction). For brevity's sake, we refer to such a system as an isotropic proton sample.

\section{A Pragmatic Interpretation}

A pragmatic way of interpreting the multiplicity of pure-state population distributions that correspond to a mixed state is to assume that each molecule does not have a defined wavefunction until the necessary measurements are made on it, and to view the density operator as the ultimate description of its physical state. Among the infinity of representations of this operator, the one that best reflects this viewpoint for the oven or the isotropic proton sample, as well as the isotropic character of these systems, is

$$
\hat{\rho}=\frac{1}{2} \hat{1},
$$

where

$$
\begin{aligned}
\hat{1}=\left|\Phi_{\alpha}\right\rangle\left\langle\Phi_{\alpha}|+|\right. & \left|\Phi_{\beta}\right\rangle\left\langle\Phi_{\beta}\right| \\
& =\left|\Phi_{+{ }_{x}}\right\rangle\left\langle\Phi_{+x}|+| \Phi_{-x}\right\rangle\left\langle\Phi_{-x}\right|
\end{aligned}
$$

is the identity operator. The lack of directionality of this makes it evident that the average component of the spin along any axis must vanish:

$$
\left\langle I_{a}\right\rangle_{\rho}=\operatorname{tr}\left(\hat{\rho} \hat{I}_{a}\right)=\frac{1}{2} \operatorname{tr} \hat{I_{a}}=0
$$

where $a$ stands for $x, y$, or $z$. According to the pragmatic interpretation, the spin direction of any particle is completely undefined-which is stronger than saying that it is unknown-before any component is measured. A measurement of, say, $I_{z}$ "creates" this property and defines a directionality in the statewhich changes to $\Phi_{\alpha}$ or $\Phi_{\beta}$-in a similar way as the act of taking a picture with a camera creates an image in its memory or its photographic film. The kind of image depends on what we have decided to photograph, as the direction appeared in the spin state depends on the orientation chosen for the measurement device.

This interpretation can be considered as an extension to the density operator of the Copenhagen interpretation of the wavefunction: as already mentioned, this states that a proton in a pure spin-state has a well-defined spin component along one, and only one, axis, while components along perpendicular axes are completely undefined; however, we are free to assume that every proton in a macroscopic sample has some spin orientation, even if we do not know which. According to the pragmatic interpretation, the proton spin would have no orientation at all until the proper measurements are made.

\section{Other Interpretations}

Are we obliged to renounce to the wavefunction as the tool for describing the states of the molecules composing a macroscopic sample? The answer is no: we can assume that each one is in a pure state, even if we do not know which. The density operator can then be considered a mathematical tool to express our incomplete knowledge about the molecular states, in which case it would describe the state of the whole sample rather than that of individual particles. Different representations of the same density operator can then be related to distinct atomic scale pictures of the sample, all of them equally compatible with our experimental information about it. This raises a new question: Is there any practical criterion for preferring one of those pictures over the others?

For the oven or the isotropic proton sample at thermal equilibrium, a valid option is to assume that half of the particles are in the pure state $\Phi_{\alpha}$ and the 
other half are in $\Phi_{\beta}$, as it is clear that a sample obtained by mixing equal parts of particles in those states would be described by the density operator [16]. However, this two-state picture is unsatisfactory from the physical point of view because it distinguishes the $z$ axis from any other direction: under isotropic conditions there is no reason for the spins to be aligned along some arbitrarily chosen axis. Of course, the same criticism applies to any other twostate picture (see the discussion preceding Eq. [17]).

The straightforward way of reconciling the isotropic character of the sample with the assignation of a pure state to every spin is to assume that these have well-defined orientations that are distributed at random among all directions. The resulting state is a mixture of a continuum of pure states with a uniform probability density (the origin of the term "density operator" is related to these kind of densities, which play a central role in classical statistics). Hence, the summation in Eq. [5] must be replaced by an integration over all orientations $\Omega=(\theta, \varphi)$, and in place of the $p_{k}$ we will have to put $p(\Omega) d \Omega=p(\theta, \varphi) \sin$ $\theta d \theta d \varphi$, where $p(\Omega)$ is the probability density per unit solid angle of spin orientations:

$$
\hat{\rho}=\int_{\theta=0}^{\pi} \int_{\varphi=0}^{2 \pi} p(\theta, \varphi) \hat{\rho}(\theta, \varphi) \sin \theta d \theta d \varphi .
$$

Here, $\hat{\rho}(\theta, \varphi)$ is the density operator representing a pure state in which the spin points to the direction $(\theta$, $\varphi)$. Its expression in terms of $\hat{I}_{z}$ eigenstates is (3)

$$
\begin{gathered}
\hat{\rho}(\theta, \varphi)=\cos ^{2} \frac{\theta}{2}\left|\Phi_{\alpha}\right\rangle\left\langle\Phi_{\alpha}\left|+\sin ^{2} \frac{\theta}{2}\right| \Phi_{\beta}\right\rangle\left\langle\Phi_{\beta}\right| \\
+\frac{1}{2} \sin \theta e^{-i \varphi}\left|\Phi_{\alpha}\right\rangle\left\langle\Phi_{\beta}\left|+\frac{1}{2} \sin \theta e^{i \varphi}\right| \Phi_{\beta}\right\rangle\left\langle\Phi_{\alpha}\right| .
\end{gathered}
$$

For a uniform mixture, the probability density is independent of the direction and it is readily evaluated from the normalization condition

$$
1=\int_{\theta=0}^{\pi} \int_{\varphi=0}^{2 \pi} p \sin \theta d \theta d \varphi=p 4 \pi \Rightarrow p=\frac{1}{4 \pi} .
$$

The density operator for the randomly oriented mixture will thus be

$$
\hat{\rho}=\int_{\theta=0}^{\pi} \int_{\varphi=0}^{2 \pi} \frac{1}{4 \pi} \hat{\rho}(\theta, \varphi) \sin \theta d \theta d \varphi .
$$

This operator can be expressed in terms of $\hat{I}_{z}$ eigenstates by introducing Eq. [23] and integrating, which leads to

$$
\hat{\rho}=\frac{1}{2}\left|\Phi_{\alpha}\right\rangle\left\langle\Phi_{\alpha}\left|+\frac{1}{2}\right| \Phi_{\beta}\right\rangle\left\langle\Phi_{\beta}\right|=\frac{1}{2} \hat{1} .
$$

Henceforth, a distribution of randomly oriented spins is physically indistinguishable from the sample obtained by mixing equal parts of $\alpha$ and $\beta$ spins, but it is a more believable picture of the particles in the oven or the isotropic proton sample. Moreover, this picture is closest to classical intuition, as its distribution of spin expectation values is identical to that of a hypothetic sample made of classically behaving magnetic moments.

This close parallelism with the classical behavior breaks down for nuclei with spin quantum number larger than $1 / 2$. Thus, although every pure state of a $\frac{1}{2}$-spin particle has an average spin vector of fixed length pointing to some direction, a 1-spin particle can be in pure states with null expected values for the spin components along the three axes; for instance, the $\hat{I}_{z}$ eigenstate with $m_{I}=0$.

The existence of different pure-state mixtures that have the same density operator is not a mere mathematical consequence of an operator having distinct representations in different basis sets, as different mixtures can be related to distinct experimental ways of preparing the sample. As already mentioned, one can produce a macroscopic sample in the state [26] by mixing equal parts of particles in the pure states $\Phi_{\alpha}$ and $\Phi_{\beta}$. These could have been previously separated with a Stern-Gerlach device oriented along the $z$ axis and, after registering $I_{z}$, the two emerging particle beams would be refocused to obtain the desired mixture. Alternatively, the whole device could be oriented along the $x$ axis so as to produce a mixture of the states $\Phi_{+x}$ and $\Phi_{-x}$. In these two samples we do know that every particle is in one of two known pure states, $\Phi_{\alpha}$ and $\Phi_{\beta}$ or $\Phi_{+x}$ and $\Phi_{-x}$, depending on the device orientation. Because both samples are described by the same density operator, they will behave identically.

In a classical statistical mixture of magnetic moments it would, in principle, be possible to measure the orientation of each moment so as to identify the microscopic composition of the mixture, but quantum indetermination prevents us from doing so in a quantum mixture. So, if we measure $I_{z}$ for a proton of a mixture with equal parts of $\Phi_{\alpha}$ and $\Phi_{\beta}$ states, there is $1 / 2$ probability of the proton being in each $\hat{I}_{z}$ eigenstate and, therefore, $1 / 2$ probability of obtaining the 
outcomes $\hbar / 2$ and $-\hbar / 2$. On the other hand, if the mixture were made of equal parts of the states $\Phi_{-x}$ and $\Phi_{+x}$, the probability would be $1 / 2$ for the two outcomes, no matter which is the state of the picked proton, because $I_{z}$ is completely indeterminate in $\hat{I}_{x}$ eigenstates. Therefore, the two mixtures lead to the same experimental results, although for different reasons. ${ }^{1}$

Likewise, we can have mixtures in which the number of pure states is larger than the wavefunction space dimension, so that the corresponding representations of the density operator are not the result of mere changes of basis. Thus, use of Eq. [23] shows that the density operator of a mixture prepared by mixing equal parts of spins oriented along three axes at $120^{\circ}$ in the $x z$ plane is

$$
\begin{aligned}
\hat{\rho}=\frac{1}{3} \hat{\rho}_{\alpha}+\frac{1}{3} \hat{\rho}\left(120^{\circ}, 0^{\circ}\right)+\frac{1}{3} \hat{\rho}\left(120^{\circ},\right. & \left.180^{\circ}\right) \\
& =\frac{1}{2} \hat{1} .
\end{aligned}
$$

Moreover, we have seen that this is also the density operator representing a mixture of a continuous infinity of equiprobable pure states.

In a sample prepared by mixing nonorthogonal pure states, there is a subtle difference between the probability of "a molecule being in one of those states" and that of "finding that particular state in a proper measurement." Thus, it is clear from Eq. [27] that in the above-considered ternary mixture, the probability of a spin being in the state $\hat{\rho}_{\alpha}$ is $1 / 3$, while Eq. [16] shows that the probability of finding the value characterizing that state in a measurement of $I_{z}$ is $1 / 2$. This does not entail any contradiction as there is a nonnull probability of obtaining the result $\hbar / 2$ in a measurement of that observable in the states $\hat{\rho}\left(120^{\circ}\right.$, $\left.0^{\circ}\right)$ and $\hat{\rho}\left(120^{\circ}, 180^{\circ}\right)$.

To grasp the meaning of the existence of different mixtures leading to the same density operator, note that a similar situation arises in a much more familiar system: white light. It is well known that light coming from the sun is a mixture of a continuous set of monochromatic waves-pure colors-covering the whole visible spectrum (and beyond). However, one

\footnotetext{
${ }^{1}$ Prof. Albert Bramon drew my attention to a subtle physical difference between these two samples: in a mixture with equal numbers of spins in the states $\Phi_{\alpha}$ and $\Phi_{\beta}$, a measurement of $I_{z}$ on every particle would give the result $\hbar / 2$ (or $-\hbar / 2$ ) exactly half of the times, while in the mixture of $\Phi_{+x}$ and $\Phi_{-x}$, the number of outcomes of, say, $\hbar / 2$ for $I_{z}$ would not necessarily be exactly half of the total. However, experimental inaccuracies would make this point difficult to verify in a macroscopic sample.
}

can also produce white light by superposing only two monochromatic beams of complementary colors; for instance, blue and yellow. This superposition is much simpler than solar light, as one could verify by passing it through a prism, but both are indistinguishable for the human eye. Indeed, there is an infinity of combinations of monochromatic beams producing white light: any pure color with its complementary, an equal-part mixture of red, green, and blue lights, etc. Similarly, there is an infinity of different mixtures of pure states that represent the same quantum mixed state, although, contrary to what happens in the case of light, the uncertainty inherent to quantum mechanics prevents us from distinguishing between two such mixtures.

\section{A SAMPLE AT EQUILIBRIUM UNDER A STATIC FIELD}

When a static magnetic field is applied along the $z$ axis $\left(\vec{B}_{0}=\vec{u}_{z} B_{0}\right)$ to a proton sample at thermodynamic equilibrium, this evolves to a new equilibrium state in which different spin orientations are no longer equivalent and, consequently, the populations of the corresponding states are no longer equal. The Hamiltonian operator for the proton spin under the magnetic field is

$$
\widehat{H_{0}}=-\hat{\vec{\mu}} \cdot \vec{B}_{0}(1-\sigma)=-\gamma \hat{I}_{z} B_{0}(1-\sigma)=\omega_{0} \hat{I}_{z},
$$

where

$$
\omega_{0} \equiv-\gamma B_{0}(1-\sigma)
$$

Its eigenfunctions - energy eigenstates or stationary pure states - are the eigenfunctions of $\hat{I}_{z}$ :

$$
\widehat{H_{0}} \Phi_{\alpha / \beta}=E_{\alpha / \beta} \Phi_{\alpha / \beta} \quad \text { with } \quad E_{\alpha / \beta}= \pm \frac{\hbar \omega_{0}}{2} .
$$

\section{A Two-State Picture}

At thermodynamic equilibrium, the sample shows no time evolution, so that Eq. [10] implies that the density operator commutes with the Hamiltonian. Hence, both operators have a complete set of common eigenfunctions, which is $\left\{\Phi_{\alpha}, \Phi_{\beta}\right\}$ for the proton sample. Then the density operator has a diagonal expression in this basis:

$$
\hat{\rho}_{B}=\rho_{\alpha \alpha}\left|\Phi_{\alpha}\right\rangle\left\langle\Phi_{\alpha}\left|+\rho_{\beta \beta}\right| \Phi_{\beta}\right\rangle\left\langle\Phi_{\beta}\right|
$$




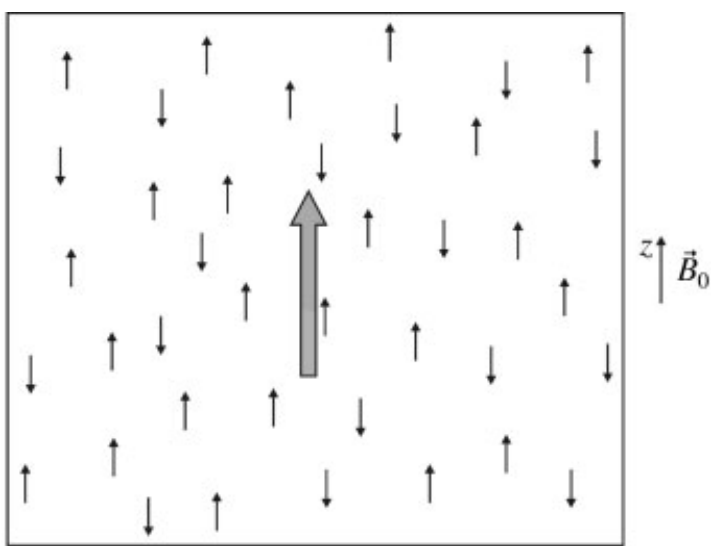

Figure 2 A two-state picture of a proton sample at equilibrium under a static magnetic field. The gray arrow represents the resultant of the spin vectors.

and the populations $\rho_{\alpha \alpha}$ and $\rho_{\beta \beta}$ are determined by the Boltzmann distribution law

$$
\rho_{\alpha \alpha / \beta \beta}=\frac{e^{-E_{\alpha / \beta} / k_{B} T}}{e^{-E_{\alpha} / k_{B} T}+e^{-E_{\beta} / k_{B} T}}=\frac{e^{\mp \hbar \omega_{0} / 2 k_{B} T}}{e^{-\hbar \omega_{0} / 2 k_{B} T}+e^{\hbar \omega_{0} / 2 k_{B} T}},
$$

where $k_{B}$ is the Boltzmann constant and $T$ the absolute temperature of the sample. In NMR experiments, $\hbar\left|\omega_{0}\right| 2 \mathrm{k}_{\mathrm{B}} \mathrm{T}$ is much less than one (typically of the order of $10^{-5}$ ), so we can safely substitute each exponential by the two first terms of its Taylor series expansion,

$$
\rho_{\alpha \alpha / \beta \beta} \approx \frac{1}{2}\left(1 \mp \frac{\hbar \omega_{0}}{2 k_{B} T}\right)=\frac{1}{2}(1 \pm \Delta p),
$$

where $\Delta p$ is the positive population difference,

$$
\Delta p \equiv \rho_{\alpha \alpha}-\rho_{\beta \beta} \equiv-\frac{\hbar \omega_{0}}{2 k_{B} T} \ll 1
$$

and the density operator takes the form

$$
\hat{\rho}_{B}=\frac{1}{2}(1+\Delta p) \hat{\rho}_{\alpha}+\frac{1}{2}(1-\Delta p) \hat{\rho}_{\beta} .
$$

Equation [34] suggests a picture of the present sample state as a mixture of molecules in the states $\Phi_{\alpha}$ and $\Phi_{\beta}$ in the proportions $(1 / 2)(1+\Delta p)$ and $(1 / 2)(1-\Delta p)$, respectively (Fig. 2). Now the $z$ axis do play a special role, so we can make no symmetrybased objection to that picture. However, there is another physical argument to reject it: if the field were gradually introduced, one would not expect that all the spins suddenly collapse into one of the $\pm z$ semi- axes at the first infinitesimal increment of field intensity. One would rather foresee a gradual distortion of a uniform distribution of orientations so as to favor the more stable states.

\section{A Quasi-Random Distribution of Spin Orientations}

Consider how the above-considered picture of an isotropic proton sample as a collection of randomly oriented spin vectors would be affected by the application of a static magnetic field. The axial symmetry of the new situation implies that the equilibrium population density $p(\theta, \varphi)$ should be independent of the angle $\varphi$. Thus, Eq. [22] for the density operator now takes the form

$$
\hat{\rho}_{B}=\int_{\theta=0}^{\pi} \int_{\varphi=0}^{2 \pi} p(\theta) \hat{\rho}(\theta, \varphi) \sin \theta d \theta d \varphi .
$$

One might be tempted to use the Boltzmann distribution law for obtaining the population density $p(\theta)$

$$
p(\theta)=\frac{e^{-\langle E\rangle_{\theta} / k_{B} T}}{Z} \approx \frac{1}{4 \pi}\left(1-\frac{\langle E\rangle_{\theta}}{k_{B} T}\right)
$$

where $\langle E\rangle_{\theta}$ is the energy expectation value of a pure state with the spin pointing to any direction, making an angle $\theta$ with the $z$ axis

$$
\begin{aligned}
\langle E\rangle_{\theta}= & \operatorname{tr}\left(\hat{\rho}(\theta, \varphi) \widehat{H_{0}}\right)=\omega_{0} \operatorname{tr}\left(\hat{\rho}(\theta, \varphi) \hat{I}_{z}\right) \\
& =\omega_{0}\left(\frac{\hbar}{2} \cos ^{2} \frac{\theta}{2}-\frac{\hbar}{2} \sin ^{2} \frac{\theta}{2}\right)=\frac{\hbar \omega_{0}}{2} \cos \theta
\end{aligned}
$$

and $Z$ (partition function) is a normalization factor:

$$
1=\int_{\theta=0}^{\pi} \int_{\varphi=0}^{2 \pi} \frac{e^{-\langle E\rangle_{\theta} / k_{B} T}}{Z} \sin \theta d \theta d \varphi \approx \frac{4 \pi}{Z} \Rightarrow Z \approx 4 \pi .
$$

The energy $\langle E\rangle_{\ominus}$ varies continuously between the two eigenvalues $E_{\alpha / \beta}$, and the population density follows a reasonable trend: the closer to the $+z$ semiaxis the spin points, the lower its energy is and the more populated the corresponding state becomes. However, substitution into Eq. [35] shows that Eq. [36] does not lead to the density operator [34], so that it must be incorrect. In fact, the 
Boltzmann formula applies only to the expression of the density operator as a mixture of pure states that are eigenstates of both, that operator and the Hamiltonian, as shown by von Neumann (4).

Although the exact form of $p(\theta)$ is not easy to deduce, one can readily verify (see Appendix A.1) that, in the hight temperature limit we are considering-for a typical NMR sample, this limit is already reached for temperatures as low as $0,1 \mathrm{~K}$-the expression for $p(\theta)$ that leads to the correct density operator is ${ }^{2}$

$$
\begin{array}{r}
p(\theta)=\frac{1}{4 \pi}\left(1-3 \frac{\langle E\rangle_{\theta}}{k_{B} T}\right)=\frac{1}{4 \pi}\left(1-3 \frac{\hbar \omega_{0}}{2 k_{B} T} \cos \theta\right) \\
=\frac{1}{4 \pi}(1+3 \Delta p \cos \theta), \quad[38]
\end{array}
$$

where $\Delta p$ is given by Eq. [33]. The physical picture bounded to Eqs. [35] and [38] for $\hat{\rho}_{B}$ is a quasirandomly oriented spin distribution with a slight preference for the directions closer to the $+z$ semiaxis.

\section{A Comparison of Both Pictures}

The representation of $\hat{\rho}_{B}$ provided by Eqs. [35] and [38] gives an intuitive picture of the spins in the proton sample that is most coherent one from the physical point of view: the random distribution of spin orientations characterizing the equilibrium state of an isolated proton sample is gradually distorted as a static magnetic field is applied so as to favor the orientations closer to the field direction (Fig. 3). Yet, we have to resort to the expression in terms of energy eigenvectors for using the Boltzmann formula to obtain the equilibrium populations (Eq. [34]). On the other hand, the latter expression is more convenient for calculation purposes. For instance, the expectation value of $I_{z}$ is readily obtained as

$$
\begin{aligned}
\left\langle I_{z}\right\rangle_{\rho B} & =\operatorname{tr}\left(\hat{\rho}_{B} \hat{I}_{z}\right)=\frac{1}{2}(1+\Delta p) \operatorname{tr}\left(\hat{\rho}_{\alpha} \hat{I}_{z}\right) \\
& +\frac{1}{2}(1-\Delta p) \operatorname{tr}\left(\hat{\rho}_{\beta} \hat{I}_{z}\right)=\frac{1}{2}(1+\Delta p)\left\langle\Phi_{\alpha}\left|\hat{I}_{z}\right| \Phi_{\alpha}\right\rangle
\end{aligned}
$$

\footnotetext{
${ }^{2}$ In a private discussion, Prof. Rodolf Tarrach made me note that the exact density operator can be expressed in terms of nonstationary pure states by substituting the Hamiltonian for the energy in the Boltzmann formula $\hat{\rho}_{B}=\int_{\theta=0}^{\pi} \int_{\varphi=0}^{2 \pi}(1 / Z) e^{-\widehat{H_{0} / 2 k_{B} T}} \hat{\rho}(\theta, \varphi) e^{-\widehat{H_{0} / 2 k_{B} T}} \sin \theta d \theta d \varphi$. However, because the resulting expression does not have the form of Eq. [35], it does not provide the desired population density $p(\theta)$.
}

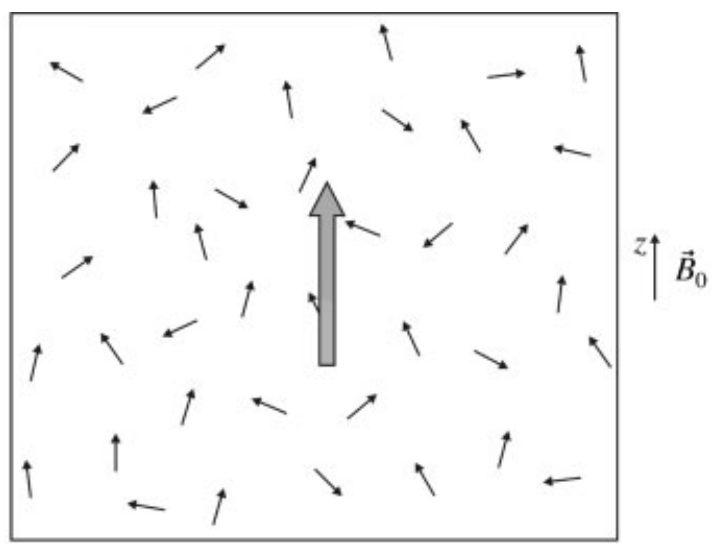

Figure 3 The quasi-random picture of a proton sample at equilibrium under a static magnetic field. The gray arrow represents the resultant of the spin vectors.

$$
\begin{array}{r}
+\frac{1}{2}(1-\Delta p)\left\langle\Phi_{\beta}\left|\hat{I}_{z}\right| \Phi_{\beta}\right\rangle=\frac{1}{2}(1+\Delta p) \frac{\hbar}{2} \\
+\frac{1}{2}(1-\Delta p)\left(-\frac{\hbar}{2}\right)=\Delta p \frac{\hbar}{2} .
\end{array}
$$

Once multiplied by the gyromagnetic ratio $\gamma$ (to convert the angular momentum into a magnetic moment) and by the number $N$ of molecules per unit volume, this expectation value gives the longitudinal macroscopic magnetization

$$
M_{z}=N \gamma\left\langle I_{z}\right\rangle_{\rho B}=N \gamma \Delta p \frac{\hbar}{2} .
$$

This result admits a straightforward interpretation in terms of the two-state picture: $M_{z}$ results from $(N /$ $2)(1+\Delta p)$ protons contributing with $\left\langle I_{z}\right\rangle=\gamma(\hbar / 2)$ and $(N / 2)(1-\Delta p)$ contributing with $-\gamma(\hbar / 2)$.

In this picture the transversal magnetization vanishes because $\left\langle I_{x}\right\rangle=\left\langle I_{y}\right\rangle=0$ for every proton (see Fig. 2):

$$
\begin{aligned}
&\left\langle I_{x / y}\right\rangle_{\rho B}=\operatorname{Tr}\left(\rho_{B} \widehat{I_{x / y}}\right)=\frac{1}{2}(1+\Delta p) \operatorname{Tr}\left(\hat{\rho}_{\alpha} \widehat{I_{x / y}}\right) \\
&+\frac{1}{2}(1-\Delta p) \operatorname{Tr}\left(\hat{\rho}_{\beta} \widehat{I_{x / y}}\right)=\frac{1}{2}(1+\Delta p)\left\langle\Phi_{\alpha}\left|\widehat{I_{x / y}}\right| \Phi_{\alpha}\right\rangle \\
&+\frac{1}{2}(1-\Delta p)\left\langle\Phi_{\beta}\left|\widehat{I_{x / y}}\right| \Phi_{\beta}\right\rangle=0 .
\end{aligned}
$$

Instead, in the quasi-randomly oriented spin distribution, the longitudinal magnetization appears as a sum of a large number of vectors pointing to every direction in space, and the transversal magnetization 
vanishes because of a cancellation of individual transversal components due to the axial symmetry of the distribution (Eq. [38]). Later, we discuss an alternative "biconical" picture frequently used in elementary discussions of NMR that mixes classical and quantum concepts.

The energy eigenstates do not play any special physical role in the most realistic quasi-randomly oriented picture. Their importance is mainly due to the practical reason of providing a diagonal expression of the equilibrium density operator in which the populations can be evaluated using the Boltzmann law.

Many other similar situations can be found in atomic and molecular physics. For instance, if we heat hydrogen atoms in an oven, the density operator describing them in equilibrium conditions at a temperature $T$, assuming that electronic spin effects are not relevant, can be written as a mixture of energy eigenstates weighed by the Boltzmann coefficients:

$$
\hat{\rho}=\sum_{n=1}^{\infty} \frac{e^{-\left(E_{n} / k_{B} T\right)}}{Z} \sum_{l=0}^{n-1} \sum_{m=-l}^{l}\left|\phi_{n l m}\right\rangle\left\langle\phi_{n l m}\right| .
$$

But there is no physical reason why the energy eigenstates $\phi_{n l m}$ should be preferred to any other stationary-e.g., $\phi_{n p_{x}}, \phi_{n p_{y}}$, etc.-or nonstationary pure states. Indeed, the individual atoms could be described by any linear combination of the wavefunctions $\phi_{n l m}$ that correspond to significantly populated states, most of which have asymmetric wavefunctions that change with time. Only if the temperature is cold enough for the ground state $\left(\phi_{1 s}\right)$ to be the only populated one we can assure that the atoms are in a pure stationary state.

\section{THE MEANING OF COHERENCES}

The nondiagonal terms of the density matrix play a crucial role in NMR experiments, and its physical interpretation deserves a careful analysis. This can be carried out from different points of view.

\section{The Relationship between Coherences and Quantum Interferences}

One of the main differences between quantum behavior and classical mechanics is the possibility of interference phenomena in material particles. It is well known that when an electron beam is splitted by a thin double slit, the resulting beams produce an interfer- ence pattern that is straightforwardly explained by the quantum laws: the wavefunction of each electron splits into two branches that rejoin behind the double slit, which can be verified by placing a detector screen:

$$
\Psi_{\text {screen }}=\frac{1}{\sqrt{2}}\left(\Phi_{\text {upper slit }}+\Phi_{\text {lower slit }}\right) .
$$

Depending on the complex relative phase of the two branches at every point of the screen their reunion can lead to different degrees of constructive or destructive interference. This translates into an increase or decrease of probability of arrival with respect to the sum of probabilities expected from classical arguments. Interestingly enough, if detectors are introduced to know the path actually followed by each electron, the interference disappears, and the probability of arrival turns out to be the sum of the probabilities observed when one slit or the other are closed. This is interpreted as a consequence of random dephasing fluctuations introduced by the detector when a particle is localized at one of the slits, and a disappearance of the other branch of the wavefunction; then there is no interference between branches, and the screen image is the result of a mixture of the states corresponding to both paths:

$$
\hat{\rho}_{\text {screen }}=\frac{1}{2}\left(\hat{\rho}_{\text {upper slit }}+\hat{\rho}_{\text {lower slit }}\right) \text {. }
$$

In the first case it is said that the interferences are due to a coherent superposition of the beams, whereas in the second there is not a definite relationship between the phases of the two beams, and we say that the superposition is incoherent.

A similar type of experiment can be done with spin-polarized particles: if a beam of neutrons, all in the spin state $\Phi_{+x}$, is split into two beams of equal intensity and the spin orientation of one of these is reversed by inserting a spin flipper, the recombination of the two beams produces neutrons in the pure state

$$
\Psi=\frac{1}{\sqrt{2}}\left(\Phi_{+x}+\Phi_{-x}\right) .
$$

By solving for $\Phi_{\alpha}$ and $\Phi_{\beta}$ in Eq. [18],

$$
\Phi_{\alpha / \beta}=\frac{1}{\sqrt{2}}\left(\Phi_{+x} \pm \Phi_{-x}\right),
$$

we see that the pure state $\Psi$ is a coherent superposition of $\Phi_{+x}$ and $\Phi_{-x}$ with the spin pointing to the $z$ 
axis $\left(\Phi_{\alpha}\right)$, as can be verified by passing the recombined beam through a Stern-Gerlach device with the field gradient aligned with that axis (5). This coherent superposition is clearly different from the incoherent mixture of $\Phi_{+x}$ and $\Phi_{-x}$ described by the density operator [17], which has null spin component along any axis (Eq. [21]).

Coherences may also appear in mixed states. Let us express the density operator $\hat{\rho}_{B}$ (Eq. [34]) in terms of $\hat{I_{x}}$ eigenvectors. This is done by using Eq. [41]:

$$
\begin{aligned}
\hat{\rho}_{B}= & \frac{1}{4}(1+\Delta p)\left|\Phi_{+x}+\Phi_{-x}\right\rangle\left\langle\Phi_{+x}+\Phi_{-x}\right| \\
& +\frac{1}{4}(1-\Delta p)\left|\Phi_{+x}-\Phi_{-x}\right\rangle\left\langle\Phi_{+x}-\Phi_{-x}\right| \\
= & \frac{1}{2}\left|\Phi_{+x}\right\rangle\left\langle\Phi_{+x}\left|+\frac{1}{2}\right| \Phi_{-x}\right\rangle\left\langle\Phi_{-x}\right| \\
& +\frac{\Delta p}{2}\left|\Phi_{+x}\right\rangle\left\langle\Phi_{-x}\left|+\frac{\Delta p}{2}\right| \Phi_{-x}\right\rangle\left\langle\Phi_{+x}\right|
\end{aligned}
$$

This expression shows up that the populations of the two states $\Phi_{ \pm x}$ in $\hat{\rho}_{B}$ are equal:

$$
P\left(I_{x}= \pm \hbar / 2\right)=\frac{1}{2},
$$

which gives a null $I_{x}$ expectation value, as happens with the density operator [17]. But while the latter represents a mixed state with zero expectation value of any spin component, $\left\langle I_{z}\right\rangle$ does not vanish for the density operator $\hat{\rho}_{B}$ (see Eq. [39]). By comparing the Eqs. [17] and [42], it follows that both operators only differ in the latter having small coherences $(\Delta p / 2)$ between $\Phi_{+x}$ and $\Phi_{-x}$.

According to Eqs. [14] and [12], coherences are weighted averages of interference terms of the pure states composing the mixture, and the averaging process may or may not completely cancel out the interferences. In $\hat{\rho}_{B}$, the averaging leaves a small amount of interference between the states $\Phi_{+x}$ and $\Phi_{-x}$ as a consequence of the nonrandomness of spin directions introduced by the magnetic field.

To state more explicitly the relationship between coherences, interferences, and spin orientations we consider an arbitrary wavefunction $\Psi$ for the proton spin expressed as a linear combination of $\Phi_{\alpha}$ and $\Phi_{\beta}$ :

$$
\Psi=c_{\alpha} \Phi_{\alpha}+c_{\beta} \Phi_{\beta}
$$

Here, $c_{\alpha}$ and $c_{\beta}$ are any complex coefficients

$$
c_{\alpha}=\left|c_{\alpha}\right| e^{i \varphi_{\alpha}} \quad c_{\beta}=\left|c_{\beta}\right| e^{i \varphi_{\beta}}
$$

subjected to the normalization condition $\left|c_{\alpha}\right|^{2}+$ $\left|c_{\beta}\right|^{2}=1$. The real numbers $\left|c_{\alpha / \beta}\right|$ are the moduli of $c_{\alpha}$ and $c_{\beta}$, and $e^{i \varphi_{\alpha / \beta}}$ are the corresponding phases. The pure state $\Psi$ can also be represented by the density operator

$$
\begin{aligned}
\hat{\rho}_{\Psi}= & |\Psi\rangle\left\langle\Psi|=| c_{\alpha} \Phi_{\alpha}+c_{\beta} \Phi_{\beta}\right\rangle\left\langle c_{\alpha} \Phi_{\alpha}+c_{\beta} \Phi_{\beta}\right| \\
= & \left|c_{\alpha}\right|^{2} \hat{\rho}_{\alpha}+\left|c_{\beta}\right|^{2} \hat{\rho}_{\beta}+c_{\alpha} c_{\beta}^{*}\left|\Phi_{\alpha}\right\rangle\left\langle\Phi_{\beta}\right| \\
& +c_{\beta} c_{\alpha}^{*}\left|\Phi_{\beta}\right\rangle\left\langle\Phi_{\alpha}\right|,
\end{aligned}
$$

with coherences

$$
\rho_{\alpha \beta}=c_{\alpha} c_{\beta}^{*} \quad \rho_{\beta \alpha}=c_{\beta} c_{\alpha}^{*} .
$$

Let us compare it with the coherent-free mixed state:

$$
\hat{\rho}=\left|c_{\alpha}\right|^{2} \hat{\rho}_{\alpha}+\left|c_{\beta}\right|^{2} \hat{\rho}_{\beta} .
$$

Although both have the same probabilities- $-\left|c_{\alpha}\right|^{2}$ and $\left|c_{\beta}\right|^{2}$ - for the outcomes $\pm \hbar / 2$ in a measurement of $I_{z}$, there are important physical differences between them. Thus, the expectation values of $I_{x}$ and $I_{y}$ for any density operator $\hat{\rho}$ depends on the coherences between $\Phi_{\alpha}$ and $\Phi_{\beta}$ :

$$
\begin{aligned}
\left\langle I_{x / y}\right\rangle_{\rho}=\operatorname{Tr}\left(\hat{\rho} \widehat{I_{x / y}}\right)=\operatorname{tr}\left[\left(\rho_{\alpha \beta}\left|\Phi_{\alpha}\right\rangle\left\langle\Phi_{\beta}\right|\right.\right. & \\
& \left.\left.+\rho_{\beta \alpha}\left|\Phi_{\beta}\right\rangle\left\langle\Phi_{\alpha}\right|\right) \widehat{I_{x / y}}\right],
\end{aligned}
$$

so that they will vanish for the incoherent mixture [47] but not for the coherent superposition [45]:

$$
\begin{array}{r}
\left\langle I_{x}\right\rangle_{\Psi}=\hbar\left|c_{\alpha}\right|\left|c_{\beta}\right| \cos \left(\varphi_{\beta}-\varphi_{\alpha}\right) \\
\left\langle I_{y}\right\rangle_{\Psi}=\hbar\left|c_{\alpha}\right|\left|c_{\beta}\right| \sin \left(\varphi_{\beta}-\varphi_{\alpha}\right) .
\end{array}
$$

This oscillatory dependence of the transversal spin components on the relative phases of the coefficients $c_{\alpha}$ and $c_{\beta}$ can be observed, for instance, in the abovementioned neutron beam experiment (5); instead of reversing the phase of one of the beams, it can be gradually changed by a phase shifter, which results in a spin polarization in complete agreement with Eqs. [49] and [50].

From Eqs. [43] and [44] it is clear that the states $\Phi_{\alpha}$ and $\Phi_{\beta}$ enter the definition of $\Psi$ with definite phases $e^{i \varphi_{\alpha}}$ and $e^{i \varphi_{\beta}}$. Although the wavefunction can be multiplied by any phase factor without changing its physical meaning, the relative phase $e^{i \varphi_{\beta}} / e^{i \varphi_{\alpha}}=$ $e^{i\left(\varphi_{\beta}-\varphi_{\alpha}\right)}$ does play a significant role: it produces the 
interference between $\Phi_{\alpha}$ and $\Phi_{\beta}$ - coherent superposition-that generates the transversal component of the spin given by Eqs. [49] and [50]. On the other hand, in the state defined by the density operator [47], the contributions of the pure states $\Phi_{\alpha}$ and $\Phi_{\beta}$ contain no information about complex phases, as $\left|c_{\alpha}\right|^{2}$ and $\left|c_{\beta}\right|^{2}$ are real positive numbers. This leads to a completely incoherent mixture of $\Phi_{\alpha}$ and $\Phi_{\beta}$.

\section{Coherences Are Dispensable}

In previous sections we have considered different expressions for certain density operators, some of them with coherences and some without. In fact, because $\hat{\rho}$ is an Hermitian operator, one can always find wavefunction basis sets for which it adopts a coherence-free diagonal form. When the density operator has degenerate eigenvalues it is evident that there is an infinity of orthonormal basis sets leading to diagonal representations (see, e.g., Eqs. [16] and [17]) but, even in the nondegenerate case, an infinity of different coherent-free expressions of the same operator exists if the orthogonality restriction is released. This kind of representations lead to a particularly simple interpretation in which one does not need to resort to quantum interferences.

Thus, one has to rely on coherences for interpreting the positive $I_{x}$ value in the pure state $\Phi_{+x}$ if one uses the representation of the corresponding density operator in terms of $\hat{I}_{z}$ eigenstates (see Eq. [18]):

$$
\begin{aligned}
\hat{\rho}_{+x}= & \left|\frac{1}{\sqrt{2}}\left(\Phi_{\alpha}+\Phi_{\beta}\right)\right\rangle\left\langle\frac{1}{\sqrt{2}}\left(\Phi_{\alpha}+\Phi_{\beta}\right)\right| \\
= & \frac{1}{2}\left|\Phi_{\alpha}\right\rangle\left\langle\Phi_{\alpha}\left|+\frac{1}{2}\right| \Phi_{\beta}\right\rangle\left\langle\Phi_{\beta}\left|+\frac{1}{2}\right| \Phi_{\alpha}\right\rangle\left\langle\Phi_{\beta}\right| \\
& +\frac{1}{2}\left|\Phi_{\beta}\right\rangle\left\langle\Phi_{\alpha}\right|,
\end{aligned}
$$

but no coherences are needed when the density operator is expressed in terms of $\hat{I}_{x}$ eigenstates:

$$
\hat{\rho}_{+x}=\left|\Phi_{+x}\right\rangle\left\langle\Phi_{+x}\right| .
$$

The opposite happens with the operator $\hat{\rho}_{B}$ (Eqs. [34] and [42]), that takes the form of a coherent-free mixture when expressed in terms of $\hat{I}_{z}$ eigenstates.

Because quantum interferences are not needed to interpret a coherent-free expression of a density operator, these kinds of representations provide atomicscale pictures closest to the classical mechanic view and, consequently, are most intuitive. This "dispensable" character of the subtle coherences, which ap- plies to any state of any quantum system, can be reassuring. However, the evolution of the density operator in time takes different forms, depending on the representation being used, and, as we shall next see, the one based on energy eigenvectors is particularly suited for such analysis. This is a crucial point in spectroscopy, where energy eigenvectors are the standard working basis.

\section{The Relationship between Coherences and Changes in Time}

Let us consider a quantum system with a time-independent Hamiltonian $\widehat{H_{0}}$. At a given time, say $t=0$, any wavefunction $\Psi_{k}(0)$ can be expanded in an orthonormal basis formed by energy eigenvectors:

$$
\Psi_{k}(0)=\sum_{j=1}^{m} c_{j k} \Phi_{j}
$$

with

$$
\widehat{H_{0}} \Phi_{j}=E_{j} \Phi_{j} \quad j=1, \ldots m
$$

This wavefunction evolves with time according to Eqs. [3] and [4], which lead to

$$
\Psi_{k}(t)=e^{-\widehat{-i t H_{0} / \hbar}} \Psi_{k}(0)=\sum_{j=1}^{m} c_{j k} e^{-i E_{j} t / \hbar} \Phi_{j} .
$$

The evolution of a mixed state that is represented at $t=0$ by the density operator

$$
\hat{\rho}(0)=\sum_{k=1}^{n} p_{k}\left|\Psi_{k}(0)\right\rangle\left\langle\Psi_{k}(0)\right|
$$

is due to the evolution of each pure state in the mixture, so that

$$
\begin{aligned}
\hat{\boldsymbol{\rho}}(t) & =\sum_{k=1}^{n} p_{k}\left|\Psi_{k}(t)\right\rangle\left\langle\Psi_{k}(t)\right| \\
& =\sum_{k=1}^{n} p_{k} \sum_{i=1}^{m} c_{i k} \sum_{j=1}^{m} c_{j k}^{*} e^{i\left(E_{j}-E_{i}\right) t / \hbar}\left|\Phi_{i}\right\rangle\left\langle\Phi_{j}\right| \\
& =\sum_{i=1}^{m} \sum_{j=1}^{m} \rho_{i j}(t)\left|\Phi_{i}\right\rangle\left\langle\Phi_{j}\right|,
\end{aligned}
$$


where (see Eq. [14])

$$
\rho_{i j}(t)=\sum_{k=1}^{n} p_{k} c_{i k} c_{j k}^{*} e^{i\left(E_{j}-E_{i}\right) t / \hbar}=\rho_{i j}(0) e^{i\left(E_{j}-E_{i}\right) t / \hbar} .
$$

The same result would be obtained by directly applying Eq. [11] to the density operator [52].

From Eq. [53] it is clear that when the density operator is expressed in an energy eigenvector basis, the populations $\rho_{i i}$ as well as coherences between degenerate states are time independent, so that there must be non-null coherences between nondegenerate states for any property to change in time.

Time evolution analysis is rather more involved if one starts from a nonstationary-state representation of the density operator. Thus, the expression in terms of energy eigenstates of the density operator $\hat{\rho}_{B}$ (Eq. [34]) clearly shows up that this operator is constant in time, since it has no coherences. However, it is not so evident how this conclusion derives from the Eq. [42] in terms of $\Phi_{ \pm x}$ : when the time-dependent expressions of these wavefunctions are introduced one finds that the four terms in the right-hand side change with time, there being an interplay between populations and coherences that results in the cancellation of the time dependence (see Appendix A.2).

\section{The Microscopic View of Time Evolution}

Let us consider a proton subjected to a static magnetic field $\vec{u}_{z} B_{0}$. If, at $t=0$, it is in the general pure state represented by Eq. [45], its initial coherences (Eq. [46])

$$
\rho_{\alpha \beta}(0)=c_{\alpha} c_{\beta}^{*} \quad \text { and } \quad \rho_{\beta \alpha}(0)=c_{\beta} c_{\alpha}^{*}
$$

evolve according to (see Eq. [53])

$$
\rho_{\alpha \beta}(t)=c_{\alpha} c_{\beta}^{*} e^{i\left(E_{\beta}-E_{\alpha}\right) t / \hbar} \text { and } \rho_{\beta \alpha}(t)=c_{\beta} c_{\alpha}^{*} e^{i\left(E_{\alpha}-E_{\beta}\right) t / \hbar} \text {. }
$$

By substituting these expressions into Eq. [48] one finds that the transversal spin components change with time according to

$$
\begin{gathered}
\left\langle I_{x}\right\rangle_{\Psi}(t)=\hbar\left|c_{\alpha}\right|\left|c_{\beta}\right| \cos \left(\varphi_{\beta}-\varphi_{\alpha}-\frac{E_{\beta}-E_{\alpha}}{\hbar} t\right) \\
\left\langle I_{y}\right\rangle_{\Psi}(t)=\hbar\left|c_{\alpha}\right|\left|c_{\beta}\right| \sin \left(\varphi_{\beta}-\varphi_{\alpha}-\frac{E_{\beta}-E_{\alpha}}{\hbar} t\right),
\end{gathered}
$$

while $\left\langle I_{z}\right\rangle_{\Psi}=\left|c_{\alpha}\right|^{2}(\hbar / 2)+\left|\mathrm{c}_{\beta}\right|^{2}(-\hbar / 2)$ remains constant. If $B_{0}=0$ then $E_{\alpha}=E_{\beta}=0$ (Eqs. [29] and
[30]) and the spin orientation keeps still, but a nonnull magnetic field breaks the energy degeneration and makes the transversal component rotate around the field with an angular velocity:

$$
-\frac{E_{\beta}-E_{\alpha}}{\hbar}=\omega_{0}=-\gamma B_{0}(1-\sigma) .
$$

It is interesting to point out that the motion represented by Eqs. [54] and [55] is identical to that obtained by applying classical dynamics to the magnetic moment, the only difference being that in the quantum treatment the spin direction has some indetermination, and it is the spin vector expectation value that precesses. This result applies to any magnetic moment proportional to an angular momentum in any pure state, which explains why classical considerations are so fruitful for explaining NMR experiments with noninteracting spins.

The evolution we have found for pure states can now be applied to any of the microscopic pictures of a proton sample under a static field. Thus, if the sample is at equilibrium, the two-state picture embodied by Eq. [34] presents every spin pointing to one of the semiaxes $+z$ and $-z$, so that no precession is produced by the magnetic field. The inexistence of a macroscopic transversal magnetization results as a consequence of all the individual spins having null transversal components at any time, because one of the coefficients $c_{\alpha}$ or $c_{\beta}$ vanishes in each of the Eqs. [54] and [55].

If the sample is viewed as a quasi-randomly oriented spin distribution, somewhat peaked toward the $+z$ semiaxis, all the spin vectors precess about the field at the same angular velocity (Eq. [56]). Because the initial phases of such rotations are distributed at random, the transversal spin components cancel out, and that cancellation remains along the time. Using a more mathematical language, the random distribution of the relative phases of the spin wavefunctions $\left(\varphi_{\beta}-\varphi_{\alpha}-\left(E_{\beta}-E_{\alpha}\right) t / \hbar\right)$ leads to null coherences in the averaging of interference terms that takes place in Eq. [53] and, therefore, to a null transversal magnetization (Eq. [48]).

\section{THE BICONICAL PICTURE}

A widely extended microscopic view of a proton sample uses some mixture of classical and quantum arguments. At equilibrium under a static magnetic field $\vec{u}_{z} B_{0}$, the spins are classed into two groups, with positive and negative $z$ component, respectively. In 


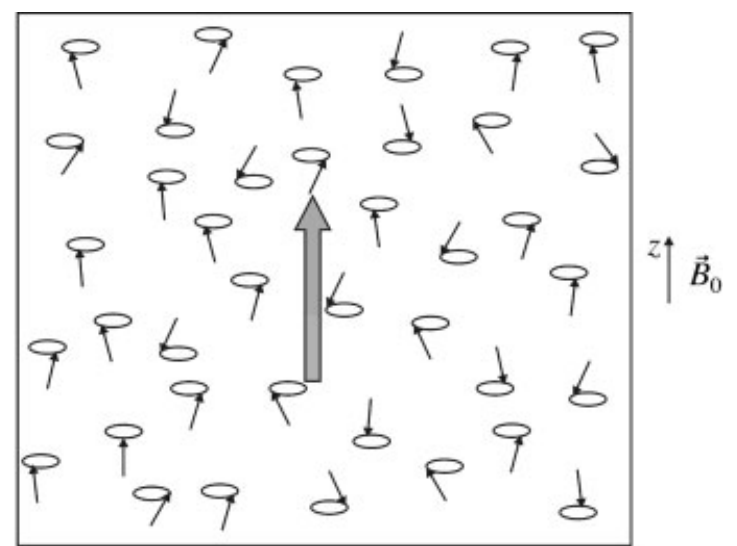

Figure 4 The biconical picture of a proton sample at equilibrium under a static magnetic field. The gray arrow represents the resultant of the spin vectors.

both of them the spin vectors are assumed to precess around the field with angular velocity $\vec{u}_{z} \omega_{0}$; that is, each vector precesses along one of two cones, as illustrated in Fig. 4. We refer to this image as "the biconical picture." The two groups of spins are associated with the energy eigenstates $\Phi_{\alpha}$ and $\Phi_{\beta}$, although this picture is incorrect from the quantum point of view. In effect, no time evolution can be observed in a quantum stationary state, and the fact that there is $1 / 2$ probability of obtaining the values $\pm \hbar / 2$ when measuring the spin component along any transversal axis means that the sign of this component is completely indeterminate, but does not imply any kind of precession. This situation is similar to that found in elementary introductions to atomic structure: one often resorts to the Bohr model for describing the motion of an electron around a nucleus as a classicallike circular orbit, but no such orbits appear in the quantum treatment; moreover, in its ground state the electron has no orbital angular momentum.

Nevertheless, we explore the possibility of exploiting the diversity of compatible pictures provided by the density operator formalism for fitting in it the biconical picture. According to this, somewhat more than half of the spins precess at a fixed angle $\theta$ and the rest do it at the angle $180^{\circ}-\theta$ at equilibrium under $\vec{u}_{z} B_{0}$. Since the spin modulus is $\hbar \sqrt{\mathrm{I}(\mathrm{I}+1)}=$ $\hbar \sqrt{3 / 4}$ and its $z$ component can only take the values $\pm \hbar / 2$, we will take $\cos \theta=( \pm \hbar / 2) /(\hbar \sqrt{3 / 4})= \pm 1 /$ $\sqrt{3}$; that is, $\theta \approx 54,7^{\circ}$ or $180^{\circ}-54,7^{\circ}=125,3^{\circ}$. The distribution of spin orientations should be uniform with respect to the angle $\varphi$, so that the density operator representing this mixture takes the form (see Eq. [35])

$$
\begin{aligned}
\hat{\rho}_{B}=\int_{0}^{2 \pi} p_{>} \hat{\rho}\left(54,7^{\circ}, \varphi\right) d \varphi \\
+\int_{0}^{2 \pi} p_{\searrow} \hat{\rho}\left(125,3^{\circ}, \varphi\right) d \varphi .
\end{aligned}
$$

One could think of determining the population densities $p_{\nearrow}$ and $p_{\searrow}$ by equating the total population of each one to the Boltzmann coefficients $\rho_{\alpha \alpha}$ and $\rho_{\beta \beta}$ (Eq. [32]):

$$
\int_{0}^{2 \pi} p_{\lambda / \triangle} d \varphi=\rho_{\alpha \alpha / \beta \beta}=\frac{1}{2}(1 \pm \Delta p),
$$

that is

$$
p_{\text {フハ }}=\frac{1}{4 \pi}(1 \pm \Delta p)
$$

but substitution into Eq. [57] shows that this does not lead to the correct density operator $\hat{\rho}_{B}$ (Eq. [34]). One could argue that the states of the precessing spins are not energy eigenstates, so that one should put the corresponding energy expectation values $\langle E\rangle_{\theta}$ (Eq. [37]) instead of the eigenvalues $E_{\alpha / \beta}$ (Eq. [30]) in the Boltzmann formula, which leads to

$$
p_{\nearrow ハ}=\frac{1}{4 \pi}(1+\Delta p \cos \theta)
$$

But, as happened in the case of the quasi-random spin distribution (see the discussion following Eq. [35]), this result does not lead to the correct expression for the density operator. As already mentioned, this is a consequence of the Boltzmann formula not being applicable to mixtures of nonstationary pure states. In Appendix A.3 we show that, now again (see Eq. [38]), a factor 3 must be introduced in order to get the correct population density:

$$
p_{\text {フハ }}=\frac{1}{4 \pi}\left(1-3 \frac{\langle E\rangle_{\theta}}{k_{B} T}\right)=\frac{1}{4 \pi}(1+3 \Delta p \cos \theta),
$$

where $\cos \theta=1 / \sqrt{3}$ for $p_{\nearrow}$ and $\cos \theta=-1 / \sqrt{3}$ for $p_{\searrow}$, that is 
$p_{\nearrow ハ}=\frac{1}{4 \pi}\left(1+3 \Delta p\left( \pm \frac{1}{\sqrt{3}}\right)\right)=\frac{1}{4 \pi}(1 \pm \sqrt{3} \Delta p)$.

Therefore, although the biconical picture is definitely the wrong image of the energy eigenstates $\Phi_{\alpha}$ and $\Phi_{\beta}$, it can be used for interpreting macroscopic proton samples provided that ad hoc expressions are taken for the population densities.

\section{THE EFFECT OF PULSES}

Let us consider again a proton sample at equilibrium under a static magnetic field $\vec{u}_{z} B_{0}$. We now discuss the interpretation of what happens when an on-resonance pulse of radiation - that is one with angular frequency $\left|\omega_{0}\right|=\gamma B_{0}(1-\sigma)$-is applied to the sample. Although NMR pulses are linearly polarized, the associated electromagnetic wave can be decomposed into two components that are circularly polarized in opposite directions and only the one with the field rotating in the same direction as the spin precession interacts with the protons, a fact that is related with angular momentum conservation (6). So we can limit our discussion to the evolution of the spins under a radiation pulse of magnetic field intensity $\vec{B}_{1}$ perpendicular to $\vec{B}_{0}$ that rotates with an angular velocity $-\gamma \vec{B}_{0}(1-\sigma)$. This evolution is best studied by moving to a reference system that rotates with that angular velocity-that is, one in which the spin vectors remain static while only $\vec{B}_{0}$ is acting. It is clear that $\vec{B}_{1}$ will also look static in that frame, so that its effect on the spins will be to make them precess about it with angular velocity $-\gamma \vec{B}_{1}$ in the rotating frame (the effect of $\sigma$ on this velocity is negligible). The total angle $\vartheta$ of precession depends on the pulse length $\tau$ :

$$
\vartheta=\left|\gamma B_{1}\right| \tau
$$

If this angle is of, say, $90^{\circ}$ and $\vec{B}_{1}$ points to the $-y$ axis of the rotating frame-in which case we will say that the pulse is applied along the $y$ axis-this will produce a $90^{\circ}$ anticlockwise rotation around this axis of every spin vector in a synchronized way.

Thus, in the two-state representation associated with the expression [34] for $\hat{\rho}_{B}$, the sample will end with somewhat more than half of the spins pointing to the $x$ axis and the rest aligned in the opposite direction (Fig. 5),

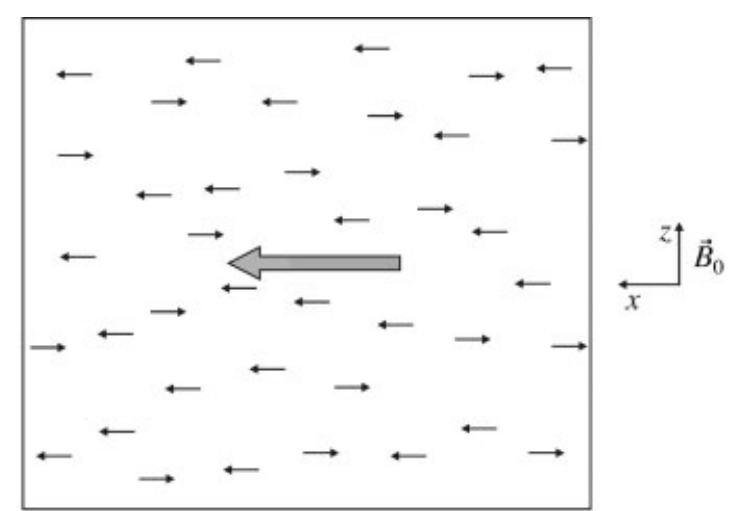

Figure 5 The result of a $90^{\circ}$ pulse applied along the $y$ axis to the two-state distribution of spins represented in Fig. 2. The gray arrow represents the resultant of the spin vectors.

$$
\hat{\boldsymbol{\rho}}_{\tau}=\frac{1}{2}(1+\Delta p) \hat{\boldsymbol{\rho}}_{+x}+\frac{1}{2}(1-\Delta p) \hat{\boldsymbol{\rho}}_{-x},
$$

leading to a macroscopic magnetization parallel to the $x$ axis. By expressing $\hat{\rho}_{ \pm x}=\left|\Phi_{ \pm x}\right\rangle\left\langle\Phi_{ \pm x}\right|$ in terms of $\Phi_{\alpha}$ and $\Phi_{\beta}$ (Eq. [18]), one sees that $\hat{\rho}_{\tau}$ is not diagonal in the energy eigenvector representation:

$$
\begin{aligned}
\hat{\rho}_{\tau}= & \frac{1}{2}(1+\Delta p)\left|\frac{1}{\sqrt{2}}\left(\Phi_{\alpha}+\Phi_{\beta}\right)\right\rangle\left\langle\frac{1}{\sqrt{2}}\left(\Phi_{\alpha}+\Phi_{\beta}\right)\right| \\
& +\frac{1}{2}(1-\Delta p)\left|\frac{1}{\sqrt{2}}\left(\Phi_{\alpha}-\Phi_{\beta}\right)\right\rangle \\
& \times\left\langle\frac{1}{\sqrt{2}}\left(\Phi_{\alpha}-\Phi_{\beta}\right)\right| \\
= & \frac{1}{2}\left|\Phi_{\alpha}\right\rangle\left\langle\Phi_{\alpha}\left|+\frac{1}{2}\right| \Phi_{\beta}\right\rangle\left\langle\Phi_{\beta}\right| \\
& +\frac{\Delta p}{2}\left|\Phi_{\alpha}\right\rangle\left\langle\Phi_{\beta}\left|+\frac{\Delta p}{2}\right| \Phi_{\beta}\right\rangle\left\langle\Phi_{\alpha}\right| .
\end{aligned}
$$

The two energy eigenstates are now equally populated, as in the isotropic sample described by the density operator [16], but there are now coherences between them that lead to a non-null transversal spin component (Eq. [48]). As found in section 6.4, the time evolution of these coherences will make the spins precess with angular velocity $\omega_{0}$ in the laboratory frame, as will the resultant macroscopic magnetization.

Likewise, the quasi-random distribution of spin orientations bounded to Eqs. [35] and [38] will also rotate around the $y$ axis so as to end with an axial symmetry about the $x$ axis and a slight preference for those directions closer to the $+x$ semiaxis (Fig. 6). 


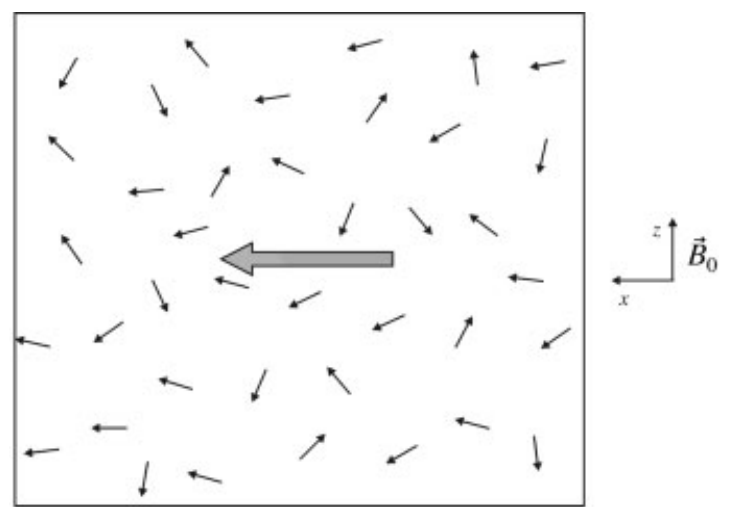

Figure 6 The result of a $90^{\circ}$ pulse applied along the $y$ axis to the quasi-randomly oriented distribution of spins represented in Fig. 3. The gray arrow represents the resultant of the spin vectors.

\section{Phase Coherence}

Within the biconical picture, the physically sound way of interpreting the effect of a $90^{\circ}$ pulse is as a $90^{\circ}$ rotation of the two conical spin distributions about the direction of the radiation magnetic field (Fig. 7). After the pulse, this spin distribution remains static in the rotating frame so that the whole bunch of vectors will precess around the static field in the laboratory frame. The latter situation does not conform to the initial biconical picture, in which all the spins vectors were precessing at one of two fixed angles with the static field direction.

To preserve the biconical picture all along the pulse, an alternative interpretation is sometimes given to its effect: instead of producing a rotation of the spins, the pulse would induce transitions between the pseudostationary states corresponding to the two cones that would lead to an equalization of their

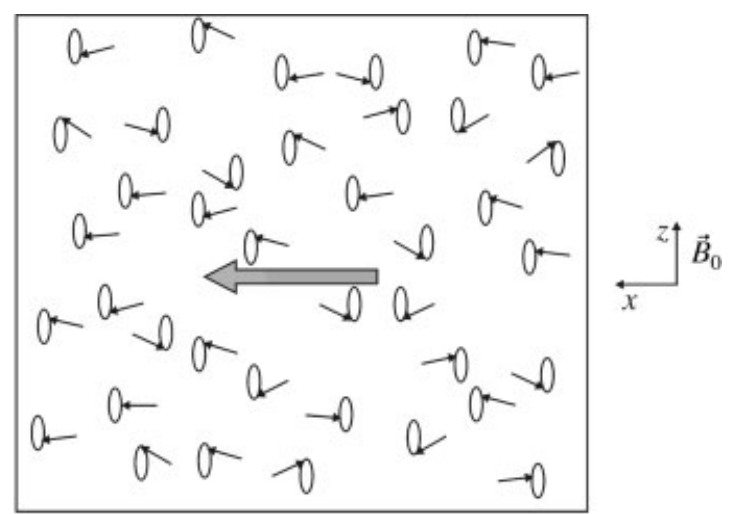

Figure 7 The result of a $90^{\circ}$ pulse applied along the $y$ axis to the biconical distribution of spins represented in Fig. 4. The gray arrow represents the resultant of the spin vectors.

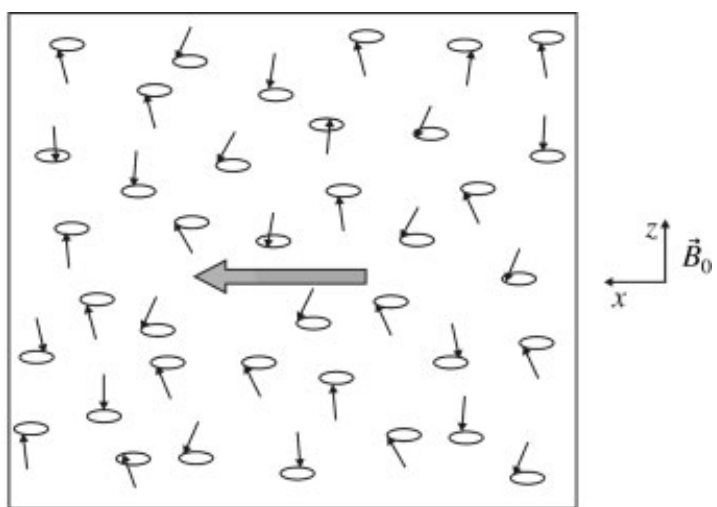

Figure 8 The result of a $90^{\circ}$ pulse applied along the $y$ axis to the biconical distribution of spins represented in Fig. 4 represented within the original biconical picture. The gray arrow represents the resultant of the spin vectors. The number of up and down spins are equal.

populations, while keeping the cones aligned with the static field. Likewise, it would modify the uniform distribution of $\varphi$ angles by grouping the spin vectors in the vicinity of $\varphi=0$, that is, around the direction of the rotating $x$ axis (Fig. 8).

This kind of evolution does not rely on any physical principle of classical or quantum electromagnetic theory: on the one hand, the effect of the radiation field is to rotate the spins about it, and, on the other, quantum transitions can only take place between the true stationary states, and the wavefunctions describing the spins of the two cones are not energy eigenfunctions. However, one could justify it inasmuch as the resulting microscopical image gives a valid description of the sample, provided that suitable population densities are chosen. In effect, substitution of the constant population densities $p_{\nearrow}$ and $p_{\searrow}$ in Eq. [57] by the ad hoc expression

$$
p_{\nearrow}=p_{\triangleleft}=\frac{1}{4 \pi}(1+\sqrt{6} \Delta p \cos \varphi)
$$

leads to the right-hand side of Eq. [60], as shown in Appendix A.4. The populations densities represented by Eq. [61] increase gradually in going from $\varphi=\pi$ to $\varphi=$ 0 , in accordance to the above-given interpretation.

We can then say that before applying the pulse, the spins were precessing with random phases about $\vec{B}_{0}$, and that the pulse induces some grouping in those phases. Comparison of Eqs. [16] or [34] with Eq. [60] shows up that such spin grouping can be related to the coherences appearing in the representation of the density operator in terms of energy eigenstates, hence, the term "phase coherence" used to refer to the nonrandomness of the precession phases. 


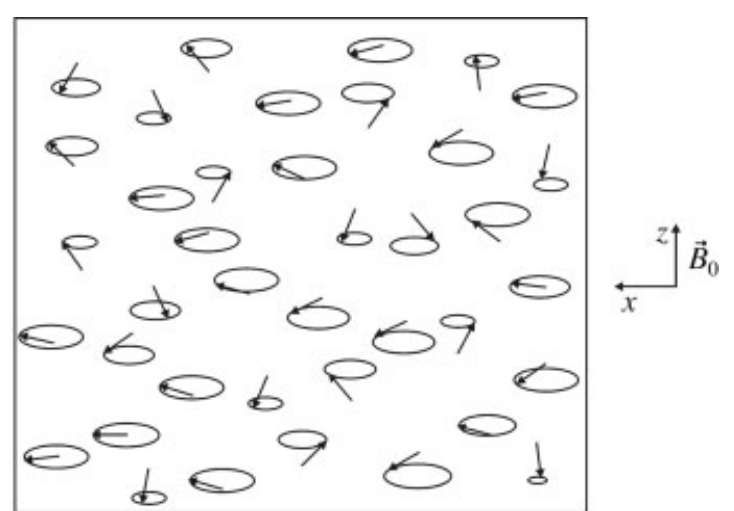

Figure 9 Free precession after a $90^{\circ}$ pulse applied along the $y$ axis of the quasi-randomly oriented distribution of spins represented in Fig. 6.

Although the validity of the interpretation of the pulse effect in terms of a grouping of precession phases can be justified by using different microscopical pictures for the sample states before and after the pulse, the fact that these are not connected by a plausible dynamics is an unpleasant feature of this description. On the other hand, the phase coherence concept arises in a more natural and straightforward way by viewing the sample as a quasi-randomly oriented spin distribution: all the spin vectors are rotated in the same way by the radiation field, so that the preferred direction of spin orientations changes from the $z$ axis to the $x$ one (see Fig. 6), then they continue their precession about the static field in the laboratory frame, all at the same angular velocity but with some grouping of the precession phases around the rotating $x$ axis (Fig. 9).

\section{Quantum Transitions}

We have discussed different ways of visualizing the effect of pulses, all of which consist of reorientations of spin vectors. However, when discussing other spectroscopic techniques, time evolution is usually presented in a different way-the electromagnetic radiation is assumed to induce discontinuous transitions between stationary states of the sample molecules. For instance, it is said that light of the proper frequency (usually in the visible or UV region) can make molecules in their ground electronic state jump into an excited electronic level.

In fact, quantum theory predicts that the effect of electromagnetic radiation on matter is a continuum evolution, independent of the frequency range of the former. Thus, when an atom in its ground state $\Phi_{1}$ is irradiated at the angular frequency $\omega_{21}=$ $\left(E_{2}-E_{1}\right) / \hbar$ of an allowed spectroscopic transition
$\Phi_{1} \rightarrow \Phi_{2}$, its wave function stops being an energy eigenstate by acquiring a gradually increasing $\Phi_{2}$ component

$$
\Psi(t=0)=\Phi_{1} \rightarrow \Psi(t)=c_{1}(t) \Phi_{1}+c_{2}(t) \Phi_{2},
$$

where $c_{1}(t)$ is a function that initially decreases from $c_{1}(0)=1$ while $c_{2}(t)$ initially increases from $c_{2}(0)=0$. The continuously evolving state $\Psi(t)$ only collapses into the state $\Phi_{2}$ if a measurement of the energy is performed that produces the corresponding eigenvalue $E_{2}$. This sudden change of quantum state involves a loss of the contribution from $\Phi_{1}$ to the wavefunction ( $c_{1}$ becomes zero) and a disappearance of the coherences between $\Phi_{1}$ to $\Phi_{2}$ present in the state just before the measurement (see Eq. [46]). This type of decoherence effect is not ruled by the Schrödinger equation, and it is the reason why two-dimensional NMR experiments cannot be performed in one go by adding to the final registration of the FID an additional measurement during the evolution time of the experiment.

In many spectroscopic techniques the typical experiment consists of a sample irradiation followed by detection, so that the sample evolution essentially reduces to quantum jumps of its constituting atoms or molecules. However, most NMR experiments involve several stages before the final registration of the FID, and no collapse takes place during those stages. Notwithstanding, one can still speak of quantum transitions in the following sense. Let us consider again a proton sample in a static field. If the density operators at equilibrium conditions $\left(\hat{\rho}_{B}\right)$ and after a $90^{\circ}$ pulse has been applied along the $y$ axis $\left(\hat{\rho}_{\tau}\right)$ are both expressed in the energy eigenvector basis (Eqs. [34] and [60]), one readily sees that the pulse has equalized the populations of $\Phi_{\alpha}$ and $\Phi_{\beta}$. Thus, if the two-state picture of the sample is adopted for both macroscopic states $-\hat{\rho}_{B}$ and $\hat{\rho}_{\tau}$-we are lead to interpret the pulse effect as a set of transitions between the pure states $\Phi_{\alpha}$ and $\Phi_{\beta}$, with a net result of $\Delta p / 2$ protons passing from the former to the latter, accompanied by the appearance of some coherence between those states.

The same population change would be produced by relaxation if an equilibrium sample is removed from the static field, but no coherence would appear in this case because of the disordered character of the relaxation mechanisms. Moreover, if coherences were initially present, as in the state $\hat{\rho}_{\tau}$, relaxation would tend to make them disappear by randomizing the relative phases that cause the quantum interferences. We thus say that the evolution originated by relaxation is incoherent by opposition to the coherent evolution induced by radiation. 


\section{OPERATOR BASIS SETS}

Just like the wavefunctions of a physical system form a vectorial space, the operators acting on those wavefunctions make up another vectorial space, usually referred to as the Liouville space. A scalar product is defined in it as follows:

$$
\langle\hat{A} \mid \hat{B}\rangle \equiv \operatorname{tr}\left(\hat{A}^{\dagger} \hat{B}\right)
$$

In fact, we have already made implicit use of operator expansions in basis sets of that space; thus, Eq. [13] is a representation of the density operator in the basis set formed by the operators

$$
\begin{aligned}
\left\{| \Phi _ { 1 } \rangle \langle \Phi _ { 1 } | , | \Phi _ { 1 } \rangle \left\langle\Phi_{2}|, \ldots|\right.\right. & \left|\Phi_{1}\right\rangle\left\langle\Phi_{m}\right|, \\
& \left.\left|\Phi_{2}\right\rangle\left\langle\Phi_{1}|, \ldots| \Phi_{m}\right\rangle\left\langle\Phi_{m}\right|\right\}
\end{aligned}
$$

which shows that the Liouville space is $m^{2}$ dimensional when the wavefunction space is $m$ dimensional. The expansion coefficients of the density operator in that basis are the elements of the density matrix (Eq. [15])

$$
\left\{\rho_{11}, \rho_{12}, \ldots \rho_{1 m}, \rho_{21}, \ldots \rho_{m m}\right\}
$$

By a suitable choice of basis in the Liouville space, the components of the density operator acquire a physical meaning that can be useful for interpreting complex NMR experiments.

\section{Hermitian Operators}

Let us consider an expansion of the density operator describing a spin system in terms of Hermitian operators $\widehat{O_{1}}, \ldots \widehat{O_{m^{2}}}$ representing relevant observables of the system

$$
\hat{\rho}=\sum_{i=1}^{m^{2}} c_{i} \widehat{O_{i}}
$$

If the basis operators are orthonormal, each coefficient of this expansion is the scalar product of the corresponding basis element by $\hat{\rho}$ :

$$
c_{i}=\left\langle\widehat{O_{i}} \mid \hat{\rho}\right\rangle=\operatorname{tr}\left(\widehat{O_{i}^{\dagger}} \hat{\rho}\right)
$$

The hermiticity of $\widehat{O_{i}}$, together with Eq. [8], can then be used to relate this coefficient to the expectation value of the observable represented by $\widehat{O_{i}}$

$$
c_{i}=\operatorname{tr}\left(\hat{\rho} \widehat{O_{i}}\right)=\left\langle O_{i}\right\rangle_{\rho} .
$$

For example, the density operator describing a proton sample at equilibrium under a static magnetic field $\vec{u}_{z} B_{0}$ (Eq. [34]) can be rewritten in the form

$$
\begin{aligned}
\hat{\rho}_{B}=\frac{1}{2}\left|\Phi_{\alpha}\right\rangle\left\langle\Phi_{\alpha}\right|+ & \frac{1}{2}\left|\Phi_{\beta}\right\rangle\left\langle\Phi_{\beta}\right| \\
& +\frac{\Delta p}{2}\left|\Phi_{\alpha}\right\rangle\left\langle\Phi_{\alpha}\left|-\frac{\Delta p}{2}\right| \Phi_{\beta}\right\rangle\left\langle\Phi_{\beta}\right| .
\end{aligned}
$$

The sum of the first two terms of this expression is the identity operator divided by 2 (Eq. [20]), and the sum of the other two is proportional to the spectral decomposition of $\hat{I}_{z}$ :

$$
\hat{I}_{z}=\frac{\hbar}{2}\left|\Phi_{\alpha}\right\rangle\left\langle\Phi_{\alpha}\left|-\frac{\hbar}{2}\right| \Phi_{\beta}\right\rangle\left\langle\Phi_{\beta}\right|
$$

so that

$$
\hat{\rho}_{B}=\frac{1}{2} \hat{1}+\frac{\Delta p}{\hbar} \hat{I}_{z}
$$

One can readily see that the four Hermitian operators

$$
\widehat{O_{1}}=\frac{1}{\sqrt{2}} \hat{1} \quad \widehat{O_{2}}=\frac{\sqrt{2}}{\hbar} \hat{I_{x}} \quad \widehat{O_{3}}=\frac{\sqrt{2}}{\hbar} \hat{I_{y}} \quad \widehat{O_{4}}=\frac{\sqrt{2}}{\hbar} \hat{I}_{z}
$$

form an orthonormal set, so that this is a basis in the Liouville space $\left(m^{2}=4\right)$ in terms of which $\hat{\rho}_{B}$ has the following expression:

$$
\hat{\rho}_{B}=\frac{1}{\sqrt{2}}\left(\frac{1}{\sqrt{2}} \hat{1}\right)+\frac{\Delta p}{\sqrt{2}}\left(\frac{\sqrt{2}}{\hbar} \hat{I}_{z}\right) .
$$

The coefficients of this representation of $\hat{\rho}_{B}$ no longer represent populations or coherences, as the basis operators are not projectors onto pure states nor crossoperators connecting pairs of pure states, as in Eq. [13]. According to Eq. [62], the coefficient $(\Delta p / \sqrt{2})$ is the expectation value of $(\sqrt{2} / \hbar) \hat{\mathrm{I}}_{\mathrm{z}}$ or, equivalently, $\Delta p(\hbar / 2)$ is the expectation value of $\hat{I}_{z}$, as previously found in a less direct way (Eq. [39]). Since $(\sqrt{2} / \hbar) \hat{I_{x}}$ and $(\sqrt{2} / \hbar) \hat{I_{y}}$ have null coefficients, $\left\langle I_{x}\right\rangle_{\rho_{B}}=\left\langle I_{y}\right\rangle_{\rho_{B}}=$ 0 (see Eq. [40]).

Likewise, the operator $\hat{\rho}_{\tau}$ describing the sample just after a $90^{\circ}$ pulse along the $y$ axis (Eq. [59]) can be put in the form 


$$
\hat{\rho}_{\tau}=\frac{1}{2} \hat{1}+\frac{\Delta p}{\hbar} \hat{I_{x}},
$$

which evidences that

$$
\left\langle I_{x}\right\rangle_{\rho_{\tau}}=\Delta p \frac{\hbar}{2} \quad\left\langle I_{y}\right\rangle_{\rho_{\tau}}=\left\langle I_{z}\right\rangle_{\rho_{\tau}}=0
$$

When this type of representation is used, the evolution of the density operator is usually obtained by first determining how each basis operator evolves under a static magnetic field and when a radiation pulse is applied; then this knowledge is used to obtain the evolution of every term in the density operator expansion.

Thus, the evolution of $\hat{\rho}_{\tau}$ (Eq. [63]) is determined by that of the identity operator, which remains always constant, and that of $\hat{I}_{x}$ (see Eq. [11]):

$$
\widehat{I_{x}} \stackrel{\hat{U}\left(t, t_{0}\right)}{\longrightarrow} \hat{U}\left(t, t_{0}\right) \widehat{I_{x}} \hat{U}^{\dagger}\left(t, t_{0}\right)
$$

When the sample is only acted by the static field $\vec{u}_{z} B_{0}$, the expression [4] for $\hat{U}$ can be used (see also Eq. [28]):

$$
\hat{U}\left(t, t_{0}\right)=e^{-i\left(t-t_{0}\right) \widehat{H_{0} / \hbar}}=e^{-i\left(t-t_{0}\right) \omega_{0} \hat{I_{z} / \hbar}}
$$

so that, taking for simplicity $t_{0}=0$,

$$
\widehat{I_{x}} \stackrel{\hat{U}(t, 0)}{\longrightarrow} e^{-i \omega_{0} t I_{z} / \hbar} \hat{I_{x}} e^{i t \omega_{0} t \hat{I}_{z} / \hbar}
$$

Taylor expansions of the exponential operators show that the preceding result can be cast into the form

$$
\widehat{I_{x}} \stackrel{\omega_{0} t}{\longrightarrow} \widehat{I_{x}} \cos \omega_{0} t+\widehat{I_{y}} \sin \omega_{0} t
$$

where, following a common practice, we have written the argument of the trigonometric functions instead of the evolution operator for indicating the type of evolution. Then, the density operator [63] evolves according to

$$
\begin{aligned}
\hat{\rho}_{\tau}(t) & =\hat{U}(t, 0)\left(\frac{1}{2} \hat{1}+\frac{\Delta p}{\hbar} \hat{I_{x}}\right) \hat{U}^{\dagger}(t, 0) \\
& =\frac{1}{2} \hat{1}+\frac{\Delta p}{\hbar} \cos \omega_{0} t \hat{I_{x}}+\frac{\Delta p}{\hbar} \sin \omega_{0} t \hat{I_{y}} .
\end{aligned}
$$

The coefficients of $\hat{I}_{x}, \hat{I}_{y}$, and $\hat{I}_{z}$ now determine the expectation values of these observables at any time $t$ :

$$
\begin{aligned}
& \left\langle I_{x}\right\rangle_{t}=\Delta p \frac{\hbar}{2} \cos \omega_{0} t=\left\langle I_{x}\right\rangle_{0} \cos \omega_{0} t \\
& \left\langle I_{y}\right\rangle_{t}=\left\langle I_{x}\right\rangle_{0} \sin \omega_{0} t \\
& \left\langle I_{z}\right\rangle_{t}=0,
\end{aligned}
$$

which shows the well-known precession of the spin vector expectation value around the $z$ axis:

$$
\left\langle I_{x}\right\rangle_{0} \vec{u}_{x} \stackrel{\omega_{0} t}{\longrightarrow}\left\langle I_{x}\right\rangle_{0}\left(\vec{u}_{x} \cos \omega_{0} t+\vec{u}_{y} \sin \omega_{0} t\right) .
$$

The parallelism between this result and Eq. [64] allows us to identify the rotation of the spin vector expectation value in the physical space with some kind of "rotation" of the corresponding operator in the Liouville space, so one can directly use the evolution equation of the latter to assess the changes experienced by the former. This provides a powerful intuitive instrument for rationalizing complex NMR experiments. It should be kept in mind, however, that it is the coefficients multiplying the normalized operators and not the operators themselves which represent expectation values.

Although, in principle, every Hermitian basis operator represents an observable, some of these may be difficult to measure. This is not the case for the basis operators we have considered for a proton sample, but in more complex systems (larger spin value or larger number of coupled spins), less-trivial operators appear in the basis sets. However, NMR devices only detect the transversal component of the magnetization, so that one usually restricts the term "observable" to that component.

In a proton sample, the expectation values of the three magnetization components together with the normalization condition $\langle\hat{\rho} \mid \hat{\rho}\rangle=1$ univocally fix the density operator, but this is no longer true for particles with a larger spin quantum number or for systems with several interacting nuclear spins. A count of the independent real parameters in the density matrix shows that one needs $m^{2}-1$ real parameters to specify the state of a system with an $m$-dimensional wavefunction space. For instance, one can use the eight real parameters $\left\langle I_{x}\right\rangle,\left\langle I_{y}\right\rangle,\left\langle I_{z}\right\rangle,\left\langle I_{x}^{2}\right\rangle,\left\langle I_{z}^{2}\right\rangle$, $\left\langle I_{x} I_{y}+I_{y} I_{x}\right\rangle,\left\langle I_{y} I_{z}+I_{z} I_{y}\right\rangle$, and $\left\langle I_{z} I_{x}+I_{x} I_{z}\right\rangle$ to fix the spin state of a 1 -spin particle. A procedure for 
measuring parameters that are quadratic in spin components is discussed in reference (7).

The expression of the density operator describing a sample with 1-spin particles at equilibrium under a static field $\vec{u}_{z} B_{0}$ is readily obtained by using the Boltzmann distribution law for the populations of the energy eigenstates $\Phi_{+z}, \Phi_{0 z}$, and $\Phi_{-z}$ :

$$
\begin{aligned}
\hat{\rho}= & p_{+z}\left|\Phi_{+z}\right\rangle\left\langle\Phi_{+z}\left|+p_{0 z}\right| \Phi_{0 z}\right\rangle\left\langle\Phi_{0 z}\left|+p_{-z}\right| \Phi_{-z}\right\rangle\left\langle\Phi_{-z}\right| \\
= & \frac{1}{3}\left(1-\frac{\hbar \omega_{0}}{k_{B} T}\right)\left|\Phi_{+z}\right\rangle\left\langle\Phi_{+z}\left|+\frac{1}{3}\right| \Phi_{0 z}\right\rangle\left\langle\Phi_{0 z}\right| \\
& +\frac{1}{3}\left(1+\frac{\hbar \omega_{0}}{k_{B} T}\right)\left|\Phi_{-z}\right\rangle\left\langle\Phi_{-z}\right|=\frac{1}{3} \hat{1}-\frac{\omega_{0}}{3 k_{B} T} \hat{I}_{z} .
\end{aligned}
$$

This expression shows up that $\left\langle I_{x}\right\rangle=\left\langle I_{y}\right\rangle=0$, while $\left\langle I_{z}\right\rangle$ is the resultant of $p_{+z}$ spins having $I_{z}=\hbar, \mathrm{p}_{0 z}$ spins having $I_{z}=0$, and $p_{-z}$ spins having $I_{z}=-\hbar$ :

$$
\begin{aligned}
\left\langle I_{z}\right\rangle=\operatorname{tr}\left(\hat{\rho} \hat{I}_{z}\right)=\frac{-\omega_{0}}{3 k_{B} T} \operatorname{tr}\left(\widehat{I}_{z}^{2}\right)=\frac{-\omega_{0}}{3 k_{B} T} 2 \hbar^{2} & \\
& =\left(p_{+z}-p_{-z}\right) \hbar .
\end{aligned}
$$

That $\left\langle I_{x}\right\rangle,\left\langle I_{y}\right\rangle$, and $\left\langle I_{z}\right\rangle$ do not determine the density operator can be corroborated by noting that any operator of the form

$$
\begin{array}{r}
\hat{\rho}=\left(a-\frac{\hbar \omega_{0}}{3 k_{B} T}\right)\left|\Phi_{+z}\right\rangle\left\langle\Phi_{+z}|+(1-2 a)| \Phi_{0 z}\right\rangle\left\langle\Phi_{0 z}\right| \\
+\left(a+\frac{\hbar \omega_{0}}{3 k_{B} T}\right)\left|\Phi_{-z}\right\rangle\left\langle\Phi_{-z}|+c| \Phi_{+z}\right\rangle\left\langle\Phi_{0 z}\right| \\
-c\left|\Phi_{0 z}\right\rangle\left\langle\Phi_{-z}|+d| \Phi_{+z}\right\rangle\left\langle\Phi_{-z}\left|+c^{*}\right| \Phi_{0 z}\right\rangle\left\langle\Phi_{+z}\right| \\
-c^{*}\left|\Phi_{-z}\right\rangle \times\left\langle\Phi_{0 z}\left|+d^{*}\right| \Phi_{-z}\right\rangle\left\langle\Phi_{+z}\right|,
\end{array}
$$

where $c$ and $d$ are arbitrary complex parameters and $a$ is a real number in the interval [0, 1/2], represents a state with the same spin-component expectation values than the state represented by Eq. [67].

\section{Non-Hermitian Operators}

When expanding the density operator in terms of operators related to observables, certain non-Hermitian ones are sometimes used. Thus, the $x$ and $y$ spin components can be combined to produce two ladder operators,

$$
\widehat{I_{ \pm}} \equiv \widehat{I_{x}} \pm i \widehat{I_{y}}
$$

that clearly are non-Hermitian:

$$
\widehat{I_{ \pm}^{\dagger}}=\widehat{I_{\mp}}
$$

By using Eq. [64] and the corresponding evolution equation for $\hat{I_{y}}$,

$$
\widehat{I_{y}} \stackrel{\omega_{0} t}{\longrightarrow} \widehat{I_{y}} \cos \omega_{0} t-\widehat{I_{x}} \sin \omega_{0} t
$$

one readily finds how the operators $\widehat{I_{ \pm}}$evolve under a static magnetic field:

$$
\widehat{I_{ \pm}} \stackrel{\omega_{0} t}{\longrightarrow} \widehat{I_{ \pm}} e^{\mp i \omega_{0} t}
$$

For positive $\gamma$ (negative $\omega_{0}$ ), this evolution consists of an increase of the phase angle with time for $\widehat{I_{+}}$and a decrease of that angle for $\widehat{I_{-}}$, both at the speed $\left|\omega_{0}\right|$, that is, a rotation on the $x y$ plane anticlockwise for $\widehat{I_{+}}$ and clockwise for $\widehat{I_{-}}$. The expectation values of $I_{x}$ and $I_{y}$ can be combined to give a complex number representing the transversal spin component

$$
\left\langle I_{x}\right\rangle+i\left\langle I_{y}\right\rangle=\left\langle I_{+}\right\rangle
$$

so that the above-mentioned parallelism between the evolution equations of operators and those of the corresponding observable expectation values could lead us to conclude that, because the spin transversal component is represented by the operator $\widehat{I_{+}}$, it should precess about the $z$ axis anticlockwise. However, Eqs. [65] and [66] show that the precession takes place clockwise for negative $\omega_{0}$. This apparent contradiction resolves by noting that, due to the non-Hermitian character of $\widehat{I_{ \pm}}$, the expectation value $\left\langle I_{+}\right\rangle$is not determined by the coefficient of $\widehat{I_{+}}$in the density operator expansion but by that of $\widehat{I_{-}}$:

$$
\left\langle I_{+}\right\rangle=\operatorname{tr}\left(\widehat{I_{+}} \hat{\rho}\right)=\operatorname{tr}\left(\widehat{I_{-}^{\dagger}} \hat{\rho}\right)=\left\langle\widehat{I_{-}} \mid \hat{\rho}\right\rangle
$$

so that it is the basis operator $\widehat{I_{-}}$that evolves like $\left\langle I_{+}\right\rangle$.

This example shows that the identification of operators in the density operator expansion with properties of the sample can be misleading, and that one should take care of choosing the expansion term leading to the proper expectation value when the corresponding operator is not Hermitian. 


\section{OTHER MOLECULAR DEGREES OF FREEDOM}

Most of the preceding discussion is essentially transferable to other molecular degrees of freedom. Consider an $\mathrm{HCl}$ gaseous sample at thermodynamic equilibrium. The internuclear molecular axes are randomly distributed, giving a null net electric polarization. The density operator describing this situation can be expressed as a mixture of rotational energy eigenstates $Y_{J M}(\theta, \varphi)$ populated according to the Boltzmann law:

$$
\hat{\rho}=\sum_{J=0}^{\infty} \sum_{M=-J}^{J} \frac{e^{-J(J+1) h B / k_{B} T}}{Z}\left|Y_{J M}\right\rangle\left\langle Y_{J M}\right| .
$$

For each state $Y_{J M}$ the probability density of nuclearaxis orientations is time independent (in an inertial reference frame translating with the molecule), so that no rotational motion shows up. On the other hand, the average rotational angular momentum points to the same direction for all those states: the arbitrarily chosen $z$ axis. Hence, the standard description of the equilibrium mixture in terms of energy eigenstates leads to a physical picture that is rather aloof from classical intuition. However, the wavefunctions of these stationary states can be combined to give nonstationary rotational wavefunctions that do represent molecules rotating around any axis in a classical-like way, except for some indetermination in the nuclearaxis orientation. Such wavefunctions provide a more familiar picture of the rotational motion than the energy eigenstates do, and an expression of the density operator in terms of them would be much more intuitive.

The case of the $\mathrm{HCl}$ vibrational motion is somewhat different. At room temperature, the great majority of $\mathrm{HCl}$ molecules are in their ground vibrational state, so that their vibrational state is pure and stationary. No time evolution can be detected in this state, and the static probability density is maximum around the nuclear equilibrium positions. This is in sharp contrast with the classical view, in which the nuclei have an oscillatory motion and spend more time at the turning points than in their equilibrium positions. Although the expectation values of the nuclear velocities are null in a reference frame that rotates with the molecule, those of the squared velocity and the kinetic vibrational energy do not vanish. This is because the quantum uncertainty of the momentum allows quantum fluctuations of this observable. The non-null squared velocity is difficult to fit together with the absence of time evolution, and this quantum picture cannot be substituted by a more intuitive, classicallike one, contrary to what happens with the rotational motion or the spin; the vibrational state being pure, there is no freedom for choosing alternative representations of the density operator.

If the sample is irradiated at the resonant frequency of the transition to the first excited vibrational state $\left(\omega=\left(E_{1}-E_{0}\right) / \hbar\right)$, the molecular state becomes nonstationary by acquiring an increasing contribution of the excited one. For weak-intensity radiation, linearly polarized along the $x$ axis, first-order time-dependent perturbation theory leads to the following expression for that contribution (for simplicity, a nonrotating molecule is considered whose internuclear axis points to the $x$ direction):

$$
c_{1}(t)=i \frac{F}{2 \hbar}\left\langle\Phi_{1}\left|\widehat{d_{x}}\right| \Phi_{0}\right\rangle t
$$

$F$ being the electric field amplitude and $\widehat{d_{x}}$ the $x$ component of the electric dipole-moment operator. Hence, the molecular state becomes

$$
\begin{aligned}
\Psi(t) & =c_{0}(t) e^{-i E_{0} t / \hbar} \Phi_{0}+c_{1}(t) e^{-i E_{1} t / \hbar} \Phi_{1} \\
& =e^{-i E_{0} t / \hbar}\left[c_{0}(t) \Phi_{0}+c_{1}(t) e^{-i \omega t} \Phi_{1}\right]
\end{aligned}
$$

where $c_{0}(t)$ decreases as $c_{1}(t)$ increases so as to keep $\Psi(t)$ normalized. A straightforward calculation shows that this wavefunction leads to a time-dependent probability density that represents an oscillation of the internuclear distance with angular frequency $\omega$, as expected on classical grounds. Moreover, the amplitude of such oscillations increases gradually with time (see Eq. [70]), which agrees with a progressive absorption of radiative energy by the sample. Such oscillations have been observed with ultrafast laser techniques (8).

The density operator corresponding to $\Psi(t)$ shows an increasing population of the excited state

$$
\begin{array}{r}
\hat{\rho}(t)=|\Psi(t)\rangle\left\langle\left.\Psi(t)|=| c_{0}(t)\right|^{2} \mid \Phi_{0}\right\rangle\left\langle\Phi_{0}\right| \\
+\left|c_{1}(t)\right|^{2}\left|\Phi_{1}\right\rangle\left\langle\Phi_{1}\left|+c_{0}(t) c_{1}^{*}(t) c^{i \omega t}\right| \Phi_{0}\right\rangle\left\langle\Phi_{1}\right| \\
+c_{0}^{*}(t) c_{1}(t) e^{-i \omega t}\left|\Phi_{1}\right\rangle\left\langle\Phi_{0}\right|,
\end{array}
$$

that is, the irradiation changes the ground vibrational state to a nonstationary one in which the probability of finding the first excited value in an energy measurement increases with time. Thus, the expression of the density operator in terms of energy eigenstates leads to the standard quantum description in terms of jumps between discrete energy levels and coherences for 
explaining the appearance of oscillations, as already found for the proton sample.

A similar discussion can be applied to electronic transitions, with the difference that now it is the electronic cloud instead of the internuclear distance that oscillates. In this case, the oscillations are too rapid to be directly observable, and the usual way of detecting the state evolution is by measuring the variation of intensity that undergoes the radiation beam on passing through the sample. Currently, individual photons can be observed, which allows us to detect energy changes on individual molecules. Such energy measurements make the wavefunction collapse from a nonstationary state similar to that of Eq. [71] to a stationary one, so that one must again resort to quantum jumps for interpreting those experimental facts.

\section{CONCLUSIONS}

The fact that there are distinct mixtures of pure states that are described by the same density operator allows us to use different physical pictures of macroscopic systems at the atomic scale. Although density operators of macroscopic samples are usually expressed as mixtures of energy eigenstates, expressions in terms of nonstationary states are equally valid-provided that ad hoc populations are attached to these statesand furnish images closer to classical intuition. The relationship between coherences of the density operator and quantum interferences hampers the use of classical pictures for interpreting those terms, but the freedom in the choice of representations always allows use of a coherent-free one that can be interpreted without having to resort to interferences.

A widespread biconical representation of a proton sample as a two-set distribution of spins that precess at a fixed angle within each set is inconsistent with classical or quantum theory, particularly with regard to the phase coherence produced by a pulse. However, the flexibility of representations admitted by the density operator allows one to find suitable pictures for the sample states before and after the pulse that, once provided with ad hoc population distributions, allow one to accommodate the biconical image into the theory. Notwithstanding, the representation of the sample as a set of spins with quasi-random orientations leads to a more natural and physically sounded interpretation of phase coherence.

The apparent discrepancy between the continuous evolution of the spin vectors in an RMN experiment and the discontinuous quantum jumps referred to when discussing other spectroscopic techniques is shown to disappear when these jumps are analyzed in the light of the strict physical meaning of the populations.

When a density operator is expanded in a basis of the Liouville space, the basis operators are usually identified with physical properties of the system, and the evolution of the latter is deduced from that of the former. The origin of such identification is analyzed to show that it can be misleading when non-Hermitian operators are used in the expansion.

Most of the preceding analysis is extendable to other spectroscopic techniques.

\section{ACKNOWLEDGMENTS}

I gratefully acknowledge insightful discussions with professors Miquel Pons and Rodolf Tarrach, suggestions of the reviewer for improving the text, and financial support from the spanish CICyT (grant codes BIO2004-05436 and CTQ2005-08459-CO2-01).

\section{APPENDIX A}

\section{The Density Operator Expressed as a Mixture of a Quasi-Randomly Oriented Distribution of Spins}

To express the density operator describing a proton sample at equilibrium under a static magnetic field as a mixture of a quasi-randomly oriented distribution of spins, we use the continuum expansion Eq. [35], where the pure state density operator $\hat{\rho}(\theta, \varphi)$ is given by Eq. [23]. For the population density $p(\theta)$, we take the ad hoc expression [38] so as to verify that it leads to the same density operator that is obtained by using the Boltzmann distribution law in an energy-eigenstate expansion:

$$
\begin{aligned}
\hat{\rho}= & \int_{\theta=0}^{\pi} \int_{\varphi=0}^{2 \pi} p(\theta) \hat{\rho}(\theta, \varphi) \sin \theta d \theta d \varphi \\
= & \int_{\theta=0}^{\pi} \int_{\varphi=0}^{2 \pi} \frac{1}{4 \pi}(1+3 \Delta p \cos \theta)\left\{\cos ^{2} \frac{\theta}{2}\left|\Phi_{\alpha}\right\rangle\left\langle\Phi_{\alpha}\right|\right. \\
& +\sin ^{2} \frac{\theta}{2}\left|\Phi_{\beta}\right\rangle\left\langle\Phi_{\beta}\left|+\frac{1}{2} \sin \theta e^{-i \varphi}\right| \Phi_{\alpha}\right\rangle\left\langle\Phi_{\beta}\right| \\
& \left.+\frac{1}{2} \sin \theta e^{i \varphi}\left|\Phi_{\beta}\right\rangle\left\langle\Phi_{\alpha}\right|\right\} \sin \theta d \theta d \varphi .
\end{aligned}
$$

The last two braked terms are seen to vanish by integrating over $\varphi$. The first two terms determine the populations of $\Phi_{\alpha}$ : 


$$
\begin{aligned}
\rho_{\alpha \alpha}= & \int_{\theta=0}^{\pi} \int_{\varphi=0}^{2 \pi} \frac{1}{4 \pi}(1+3 \Delta p \cos \theta) \cos ^{2} \frac{\theta}{2} \sin \theta d \theta d \varphi \\
= & \frac{1}{4} \int_{\theta=0}^{\pi}(1+3 \Delta p \cos \theta)(1+\cos \theta) \sin \theta d \theta \\
= & \frac{1}{4} \int_{\theta=0}^{\pi}[\sin \theta+\cos \theta \sin \theta+3 \Delta p(\cos \theta \sin \theta \\
& \left.\left.+\cos ^{2} \theta \sin \theta\right)\right] d \theta=\frac{1}{2}(1+\Delta p)
\end{aligned}
$$

and $\Phi_{\beta}$ :

$$
\begin{aligned}
\rho_{\beta \beta} & =\int_{\theta=0}^{\pi} \int_{\varphi=0}^{2 \pi} \frac{1}{2}(1+3 \Delta p \cos \theta) \sin ^{2} \frac{\theta}{2} \sin \theta d \theta d \varphi \\
& =\frac{1}{2}(1-\Delta p) .
\end{aligned}
$$

Comparison with Eq. [34] shows that those populations correspond to the density operator $\hat{\rho}_{B}$.

\section{Evolution of the Density Operator Expressed in Terms of Nonstationary States}

To obtain the evolution of the density operator describing a proton sample at equilibrium under a static magnetic field $\left(\hat{\rho}_{B}\right)$ from its expression in terms of the nonstationary wavefunctions $\Phi_{ \pm x}$ (see Eq. [42]), we first express these in terms of energy eigenstates (Eq. [18]) and make use of Eq. [51]:

$$
\begin{aligned}
\Phi_{ \pm x}(t)=\frac{1}{\sqrt{2}}\left(e^{-i E_{\alpha} / \hbar t} \Phi_{\alpha}\right. & \left. \pm e_{\beta}^{-i E_{\beta} / \hbar t} \Phi_{\beta}\right) \\
& =\frac{1}{\sqrt{2}}\left(e^{-i \omega_{0} / 2 t} \Phi_{\alpha} \pm e^{i \omega_{0} / 2 t} \Phi_{\beta}\right) .
\end{aligned}
$$

Substitution of this result into Eq. [42] gives

$$
\begin{aligned}
& \hat{\rho}_{B}(t)=\frac{1}{4}\left|e^{-i \omega_{0} / 2 t} \Phi_{\alpha}+e_{\beta}^{i \omega_{0} / 2 t} \Phi_{\beta}\right\rangle\left\langle e^{-i \omega_{0} / 2 t} \Phi_{\alpha}+e^{i \omega_{0} / 2 t} \Phi_{\beta}\left|+\frac{1}{4}\right| e^{-i \omega_{0} / 2 t} \Phi_{\alpha}-e^{i \omega_{0} / 2 t} \Phi_{\beta}\right\rangle\left\langle e^{-i \omega_{0} / 2 t} \Phi_{\alpha}-e^{i \omega_{0} / 2 t} \Phi_{\beta}\right| \\
&+\frac{\Delta p}{4}\left|e^{-i \omega_{0} / 2 t} \Phi_{\alpha}+e^{i \omega_{0} / 2 t} \Phi_{\beta}\right\rangle\left\langle e^{-i \omega_{0} / 2 t} \Phi_{\alpha}-e^{i \omega_{0} / 2 t} \Phi_{\beta}\left|+\frac{\Delta p}{4}\right| e^{-i \omega_{0} / 2 t} \Phi_{\alpha}-e^{i \omega_{0} / 2 t} \Phi_{\beta}\right\rangle\left\langle e^{-i \omega_{0} / 2 t} \Phi_{\alpha}+e^{i \omega_{0} / 2 t} \Psi_{\beta}\right| \\
&=\frac{1}{4}\left(\left|\Phi_{\alpha}\right\rangle\left\langle\Phi_{\alpha}|+| \Phi_{\beta}\right\rangle\left\langle\Phi_{\beta}\left|+e^{-i \omega_{0} / t}\right| \Phi_{\alpha}\right\rangle\left\langle\Psi_{\beta}\left|+e^{i \omega_{0} / t}\right| \Phi_{\beta}\right\rangle\left\langle\Phi_{\alpha}\right|\right)+\frac{1}{4}\left(\left|\Phi_{\alpha}\right\rangle\left\langle\Phi_{\alpha}|+| \Phi_{\beta}\right\rangle\left\langle\Phi_{\beta}\left|-e^{-i \omega_{0} / t}\right| \Phi_{\alpha}\right\rangle\left\langle\Phi_{\beta}\right|\right. \\
&\left.-e^{i \omega_{0} / t \mid}\left|\Phi_{\beta}\right\rangle\left\langle\Phi_{\alpha}\right|\right)+ \frac{\Delta p}{4}\left(\left|\Phi_{\alpha}\right\rangle\left\langle\Phi_{\alpha}|-| \Phi_{\beta}\right\rangle\left\langle\Phi_{\beta}\left|-e^{-i \omega_{0} / t}\right| \Phi_{\alpha}\right\rangle\left\langle\Phi_{\beta}\left|+e^{i \omega_{0} / t}\right| \Phi_{\beta}\right\rangle\left\langle\Phi_{\alpha}\right|\right)+\frac{\Delta p}{4}\left(\left|\Phi_{\alpha}\right\rangle\left\langle\Phi_{\alpha}|-| \Phi_{\beta}\right\rangle\left\langle\Phi_{\beta}\right|\right. \\
&\left.+e^{-i \omega_{0} / t}\left|\Phi_{\alpha}\right\rangle\left\langle\Phi_{\beta}\left|-e^{i \omega_{0} / t}\right| \Phi_{\beta}\right\rangle\left\langle\Phi_{\alpha}\right|\right)=\frac{1}{2}(1+\Delta p)\left|\Phi_{\alpha}\right\rangle\left\langle\Phi_{\alpha}\left|+\frac{1}{2}(1-\Delta p)\right| \Phi_{\beta}\right\rangle\left\langle\Phi_{\beta}\right|=\hat{\rho}_{B}(0) .
\end{aligned}
$$

This shows that the density operator remains constant because of a cancellation of time-dependent terms.

\section{The Biconical Representation of the Density Operator}

For a proton sample at equilibrium under a static magnetic field, the density operator representing the biconical spin distribution introduced in section 7 is given by Eq. [57]. The pure-state density operators $\hat{\rho}\left(54,7^{\circ}, \varphi\right)$ and $\hat{\rho}\left(125,3^{\circ}, \varphi\right)$ can be expressed in terms of $\Phi_{\alpha}$ and $\Phi_{\beta}$ by using Eq. [23] together with the results

$$
\begin{gathered}
\sin 54,7^{\circ}=\sin 125,3^{\circ}=\sqrt{\frac{2}{3}} \\
\cos ^{2} \frac{54,7^{\circ}}{2}=\frac{\sqrt{3}+1}{2 \sqrt{3}} \quad \sin ^{2} \frac{54,7^{\circ}}{2}=\frac{\sqrt{3}-1}{2 \sqrt{3}} \\
\cos ^{2} \frac{125,3^{\circ}}{2}=\frac{\sqrt{3}-1}{2 \sqrt{3}} \sin ^{2} \frac{125,3^{\circ}}{2}=\frac{\sqrt{3}+1}{2 \sqrt{3}},
\end{gathered}
$$


that is,

$$
\begin{aligned}
\hat{\rho}\left(54,7^{\circ}, \varphi\right)=\frac{\sqrt{3}+1}{2 \sqrt{3}}\left|\Phi_{\alpha}\right\rangle\left\langle\Phi_{\alpha}\right| & \\
+\frac{\sqrt{3}-1}{2 \sqrt{3}}\left|\Phi_{\beta}\right\rangle\left\langle\Phi_{\beta}\right| & +\frac{1}{\sqrt{6}} e^{-i \varphi}\left|\Phi_{\alpha}\right\rangle\left\langle\Phi_{\beta}\right| \\
& +\frac{1}{\sqrt{6}} e^{i \varphi}\left|\Phi_{\beta}\right\rangle\left\langle\Phi_{\alpha}\right|
\end{aligned}
$$

$$
\begin{aligned}
\hat{\rho}\left(125,3^{\circ}, \varphi\right) & =\frac{\sqrt{3}-1}{2 \sqrt{3}}\left|\Phi_{\alpha}\right\rangle\left\langle\Phi_{\alpha}\right| \\
+ & \frac{\sqrt{3}+1}{2 \sqrt{3}}\left|\Phi_{\beta}\right\rangle\left\langle\Phi_{\beta}\right| \\
+ & \frac{1}{\sqrt{6}} e^{-i \varphi}\left|\Phi_{\alpha}\right\rangle\left\langle\Phi_{\beta}\right| \\
& +\frac{1}{\sqrt{6}} e^{i \varphi}\left|\Phi_{\beta}\right\rangle\left\langle\Phi_{\alpha}\right| .
\end{aligned}
$$

Substitution into Eq. [57] of these expressions together with the ad hoc population densities [58] leads to Eq. [34] for $\hat{\rho}_{B}$ :

$$
\begin{aligned}
& \int_{0}^{2 \pi} p_{\nearrow} \hat{\rho}\left(54,7^{\circ}, \varphi\right) d \varphi+\int_{0}^{2 \pi} p_{\searrow} \hat{\rho}\left(125,3^{\circ}, \varphi\right) d \varphi=\frac{1}{4 \pi}(1+\sqrt{3} \Delta p) \int_{0}^{2 \pi}\left\{\frac{\sqrt{3}+1}{2 \sqrt{3}}\left|\Phi_{\alpha}\right\rangle\left\langle\Phi_{\alpha}\left|+\frac{\sqrt{3}-1}{2 \sqrt{3}}\right| \Phi_{\beta}\right\rangle\left\langle\Phi_{\beta}\right|\right. \\
& +\frac{1}{\sqrt{6}} e^{-i \varphi}\left|\Phi_{\alpha}\right\rangle\left\langle\Phi_{\beta}\left|+\frac{1}{\sqrt{6}} e^{i \varphi}\right| \Phi_{\beta}\right\rangle\left\langle\Phi_{\alpha}|| d \varphi+\frac{1}{4 \pi}(1-\sqrt{3} \Delta p) \int_{0}^{2 \pi}\left\{\frac{\sqrt{3}-1}{2 \sqrt{3}}\left|\Phi_{\alpha}\right\rangle\left\langle\Phi_{\alpha}\left|+\frac{\sqrt{3}+1}{2 \sqrt{3}}\right| \Phi_{\beta}\right\rangle\left\langle\Phi_{\beta}\right|\right.\right. \\
& \left.+\frac{1}{\sqrt{6}} e^{-i \varphi}\left|\Phi_{\alpha}\right\rangle\left\langle\Phi_{\beta}\left|+\frac{1}{\sqrt{6}} e^{i \varphi}\right| \Phi_{\beta}\right\rangle\left\langle\Phi_{\alpha}\right|\right\} d \varphi=\left[(1+\sqrt{3} \Delta p) \frac{\sqrt{3}+1}{4 \sqrt{3}}+(1-\sqrt{3} \Delta p) \frac{\sqrt{3}-1}{4 \sqrt{3}}\right]\left|\Phi_{\alpha}\right\rangle\left\langle\Phi_{\alpha}\right| \\
& +\left[(1+\sqrt{3} \Delta p) \frac{\sqrt{3}-1}{4 \sqrt{3}}+(1-\sqrt{3} \Delta p) \frac{\sqrt{3}+1}{4 \sqrt{3}}\right]\left|\Phi_{\beta}\right\rangle\left\langle\Phi_{\beta}\left|=\frac{1}{2}(1+\Delta p)\right| \Phi_{\alpha}\right\rangle\left\langle\Phi_{\alpha}\right| \\
& +\frac{1}{2}(1-\Delta p)\left|\Phi_{\beta}\right\rangle\left\langle\Phi_{\beta}\right|=\hat{\rho}_{B} .
\end{aligned}
$$

\section{The Biconical Representation of the} Density Operator After a $90^{\circ}$ Pulse

To see whether the biconical picture is a valid representation of the density operator describing a proton sample after a $90^{\circ}$ pulse has been applied along the $y$ axis, we introduce the ad hoc population density [61] together with the pure-state density operators [72] and [73] into the density operator expansion [57]:

$$
\begin{aligned}
\hat{\rho}= & \int_{0}^{2 \pi} p_{>} \hat{\rho}\left(54,7^{\circ}, \varphi\right) d \varphi+\int_{0}^{2 \pi} p_{\searrow} \hat{\rho}\left(125,3^{\circ}, \varphi\right) d \varphi=\int_{0}^{2 \pi} \frac{1}{4 \pi}(1+\sqrt{6} \Delta p \cos \varphi)\left\{\frac{\sqrt{3}+1}{2 \sqrt{3}}\left|\Phi_{\alpha}\right\rangle\left\langle\Phi_{\alpha}\right|\right. \\
& \left.+\frac{\sqrt{3}-1}{2 \sqrt{3}}\left|\Phi_{\beta}\right\rangle\left\langle\Phi_{\beta}\left|+\frac{1}{\sqrt{6}} e^{-i \varphi}\right| \Phi_{\alpha}\right\rangle\left\langle\Phi_{\beta}\left|+\frac{1}{\sqrt{6}} e^{i \varphi}\right| \Phi_{\beta}\right\rangle\left\langle\Phi_{\alpha}\right|\right\} d \varphi+\int_{0}^{2 \pi} \frac{1}{4 \pi}(1+\sqrt{6} \Delta p \cos \varphi)\left\{\frac{\sqrt{3}-1}{2 \sqrt{3}}\left|\Phi_{\alpha}\right\rangle\left\langle\Phi_{\alpha}\right|\right.
\end{aligned}
$$




$$
\begin{array}{r}
\left.+\frac{\sqrt{3}+1}{2 \sqrt{3}}\left|\Phi_{\beta}\right\rangle\left\langle\Phi_{\beta}\left|+\frac{1}{\sqrt{6}} e^{-i \varphi}\right| \Phi_{\alpha}\right\rangle\left\langle\Phi_{\beta}\left|+\frac{1}{\sqrt{6}} e^{i \varphi}\right| \Phi_{\beta}\right\rangle\left\langle\Phi_{\alpha}\right|\right\} d \varphi=\frac{1}{2}\left|\Phi_{\alpha}\right\rangle\left\langle\Phi_{\alpha}\left|+\frac{1}{2}\right| \Phi_{\beta}\right\rangle\left\langle\Phi_{\beta}\right|+\frac{\Delta p}{2 \pi} \int_{0}^{2 \pi}\left(\cos ^{2} \varphi\right. \\
-i \cos \varphi \sin \varphi) d \varphi\left|\Phi_{\alpha}\right\rangle\left\langle\Phi_{\beta}\left|+\frac{\Delta p}{2 \pi} \int_{0}^{2 \pi}\left(\cos ^{2} \varphi+i \cos \varphi \sin \varphi\right) d \varphi\right| \Phi_{\beta}\right\rangle\left\langle\Phi_{\alpha}\left|=\frac{1}{2}\right| \Phi_{\alpha}\right\rangle\left\langle\Phi_{\alpha}\left|+\frac{1}{2}\right| \Phi_{\beta}\right\rangle\left\langle\Phi_{\beta}\right| \\
+\frac{\Delta p}{2}\left|\Phi_{\alpha}\right\rangle\left\langle\Phi_{\beta}\left|+\frac{\Delta p}{2}\right| \Phi_{\beta}\right\rangle\left\langle\Phi_{\alpha}\right| .
\end{array}
$$

Equation [60] shows that this result equals $\hat{\rho}_{\tau}$.

\section{REFERENCES}

1. Levitt MH. 2001. Spin dynamics. Basics of nuclear magnetic resonance. Chichester, England: Wiley.

2. Aspect A, Dalibard J, Roger G. 1982. Experimental tests of Bell's inequalities using time-varying analyzers. Phys Rev Lett 49:1804-1807.

3. Cohen-Tannoudji C, Diu B, Laloë F. 1986. Mécanique quantique. Paris: Hermann. Chap. IV, Eq. [A-22-a].

4. von Neumann J. 1955. Mathematical foundation of quantum mechanics. New Jersey: Princeton U. P.; §V. 3.

5. Summhammer J, Badurek G, Rauch H, Kischko U. 1982. Explicit experimental verification of quantum spin-state superposition. Phys Lett A 90:110-112.

6. Paniagua JC, Moyano A. 1996. On the way of introducing some basic NMR aspects. From the classical and naïve quantum models to the density-operator approach. J Chem Ed 73:310-318.
7. Ballentine LE. 1998. Quantum mechanics. A modern development. Singapore: World Scientific. p 213-214.

8. Zewail AH. 2000. Femtochemistry: atomic-scale dynamics of the chemical bond. J Phys Chem A 104:56605694.

\section{BIOGRAPHY}

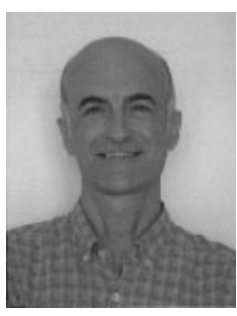

Juan Carlos Paniagua studied chemistry at the University of Barcelona and read his Ph.D. thesis in 1984 in the Physical Chemistry Department in which he teaches quantum chemistry and spectroscopy as an associate professor. Although his research has mainly developed in the field of quantum chemistry, he has always been concerned with the interaction of radiation with matter, especially in what refers to the physical interpretation of the formalism. Now his interest is focused on NMR and its amazing power to check and illustrate the quantum behavior of matter. 